\title{
Asymptotic analysis of mode-coupling theory of active nonlinear microrheology
}

\author{
M. V. Gnann ${ }^{1,2}$ and Th. Voigtmann ${ }^{2,3,4}$ \\ ${ }^{1}$ Max Planck Institute for Mathematics in the Sciences, 04103 Leipzig, Germany \\ ${ }^{2}$ Fachbereich Physik, Universität Konstanz, 78457 Konstanz, Germany \\ ${ }^{3}$ Zukunftskolleg, Universität Konstanz, 78457 Konstanz, Germany \\ ${ }^{4}$ Institut für Materialphysik im Weltraum, Deutsches Zentrum für Luft- und Raumfahrt (DLR), 51170 Köln, Germany
}

(Dated: March 25, 2021)

\begin{abstract}
We discuss a schematic model of mode-coupling theory for force-driven active nonlinear microrheology, where a single probe particle is pulled by a constant external force through a dense host medium. The model exhibits both a glass transition for the host, and a force-induced delocalization transition, where an initially localized probe inside the glassy host attains a nonvanishing steady-state velocity by locally melting the glass. Asymptotic expressions for the transient density correlation functions of the schematic model are derived, valid close to the transition points. There appear several nontrivial time scales relevant for the decay laws of the correlators. For the nonlinear friction coefficient of the probe, the asymptotic expressions cause various regimes of power-law variation with the external force, and two-parameter scaling laws.
\end{abstract}

PACS numbers: 82.70.-y 64.70.pv 83.10.-y

\section{INTRODUCTION}

Microrheology is a modern technique that allows to probe complex fluids on mesoscopic length scales. One inserts a probe particle, typically $\mu \mathrm{m}$-sized, into a host liquid of constituents that are roughly of the same size (such as colloidal dispersions or biophysical fluids). Monitoring the motion of the probe, one can infer local viscoelastic response functions of the host liquid. A particularly compelling extension of the technique is called active microrheology: here, the probe is subjected to a controlled external drive. This is most conveniently applied by using laser tweezers or magnetically susceptible probe particles, or even by tailoring colloidal probes such that they undergo self-driven motion due to chemical processes [1]. Active microrheology has become a major tool in biophysics and for colloidal model systems [4 7].

Here we focus on force-driven active microrheology, where a constant external force $\underline{F}_{\text {ex }}$ is applied to the probe particle. A natural quantity to observe then is the resulting probe velocity $\underline{v}$, and specifically its ensembleaveraged stationary value, $\langle\underline{v}\rangle_{t \rightarrow \infty}$. The influence of the host liquid is characterized by a friction coefficient,

$$
\zeta\langle\underline{v}\rangle_{t \rightarrow \infty}=\underline{F}_{\mathrm{ex}} .
$$

For typical soft-matter systems, thermal fluctuations give rise to forces in the range of $\mathrm{pN}$; it is hence easy to drive the system into the nonlinear nonequilibrium regime using active microrheology. In Eq. (1), the friction coefficient $\zeta\left(F_{\mathrm{ex}}\right)$ then becomes a function of the applied force. This makes analysis of the experiment vastly more difficult, since one needs to employ a theory of the nonlinear probe-bath interactions. If that is available, the technique can, however, be rewarding, as it gives access to much more information about the complex host liquid than a linear-response setup would. Also, compared to other nonlinear-response techniques, such as macroscopic rheology, one gains access to the microscopic mechanisms relevant for the dynamics 8 .

Recently, a microscopic theory for the force-driven active nonlinear microrheology has been proposed [9], using a combination of the integration-through transients (ITT) scheme together with approximations inspired by the mode-coupling theory for the glass transition (MCT). ITT expresses the nonlinear friction coefficient $\zeta\left(F_{\text {ex }}\right)$ via a relation of (generalized) Green-Kubo type, through a transient force autocorrelation function. This is a correlation function taken with the equilibrium ensemble, but the full nonequilibrium dynamics. Governed by the idea that in a dense system, structural relaxation via density fluctuations is the dominant slow dynamical process, MCT provides an approximate closure for this correlation function in terms of (again, transient) density correlators. These are calculated by a set of nonlinear integro-differential equations. As a result, the nontrivial relaxation pattern predicted for the density correlators directly gives rise to nonlinearities in the friction coefficient.

So far, the full MCT-ITT equations for active microrheology have proven not amenable to extended numerical treatment. The strategy used in Ref. 9 therefore was to implement an ad-hoc simplified model that reduces the complexity down to a single fluctuation mode in the hope of retaining all nontrivial mathematical features of the original set of equations. Introducing a few adjustable parameters to this so-called schematic model, a successful quantitative analysis of available computersimulation and experimental data provides an ex posteriori justification for doing so. The original schematic model proved too restrictive in certain aspects. Recently, an improved version of the model has been presented [10, which allows convincing fits of the data in all accessible regimes by taking into account some aspects of the forceinduced spatial anisotropy in the dynamics.

The data show a strong nonlinearity in $\zeta\left(F_{\mathrm{ex}}\right)$ : in a 
relatively narrow range of forces, close to the glass transition the friction coefficient drops by orders of magnitude, separating a near-equilibrium low-force regime from a high-force regime. The schematic MCT models interpret the resulting strong drop as a precursor to a probe-delocalization transition: inside a glassy host, the probe particle is held in a nearest-neighbor cage (formally $\zeta \rightarrow \infty$ at low forces, for the ideal-glass case) that can sustain a finite amount of external force. Once the applied force exceeds a critical threshold $F_{\mathrm{ex}}^{\mathrm{c}}$, the probe's nearest-neighbor cage is forced open so that a finite mean velocity results (and $\zeta$ drops to a finite value). The threshold force is thus interpreted as a measure of the cage strength. Since cages are formed in a highly collective process involving the host particles and their local structure, active nonlinear microrheology is in principle a unique tool to probe the local rigidity of that structure. In fact, $F_{\mathrm{ex}}^{\mathrm{c}}=\mathcal{O}\left(50 k_{\mathrm{B}} T / \sigma\right)$ has been measured [9], greatly exceeding the typical force scale of thermal fluctuations, $k_{\mathrm{B}} T / \sigma$ (here, $\sigma$ is a typical host-particle size).

In order to understand the asymptotic behavior of $\zeta\left(F_{\text {ex }}\right)$ close to the critical force within ITT-MCT, the asymptotic behavior of the correlation functions close to this delocalization transition has to be understood. In essence, a two-parameter scaling prescription is sought for, since one is dealing with both the distance to the glass transition, and the distance to the delocalization transition, as small parameters.

In this paper, we present an asymptotic treatment of the schematic ITT-MCT equations for force-driven active nonlinear rheology. We discuss the set of equations that has been solved numerically in Ref. [10, where the model was shown to describe both computer simulation and experiment quantitatively. The calculation proceeds by considering the double limit of approaching the glass transition and the delocalization transition, which yields two small parameters and various asymptotic results depending on their ratio and sign. The techniques we use are similar to those that have been used earlier to derive different two-parameter scaling laws within MCT, e.g., for the extended mode-coupling theory including a schematic hopping term [11, or for the ITT-MCT equations describing the macrorheology for a given constant shear rate [12].

Our analysis, however, has aspects that differ from the above-mentioned cases. Due to the nonequilibrium nature of the problem, the time-evolution operator is nonHermitian, and gives rise to complex-valued correlation functions. Usually, in the regime of structural relaxation, one can safely assume the correlation functions appearing in the theory to be real-valued and completely monotone; this holds rigorously for overdamped short-time dynamics as applicable to colloidal suspensions in equilibrium [13, 14. These properties are used in the asymptotic expansion, for example to ensure that the singularities to be discussed belong to a certain class of bifurcations [15. Even in the macro-rheology of colloidal suspensions, where external flow is represented by a non-Hermitian generalized Smoluchowski operator, taking into account the mechanism of shear advection separately allows one to return to real-valued correlation functions and to a scheme of asymptotic expansions that closely mirrors the one followed in equilibrium [16 18].

In the equations for active microrheology, some assumptions entering the standard discussion of MCT are no longer valid. Consequently, the mathematical classification of the transition between localized and delocalized probe particles (in the idealized glass) is still open. We restrict ourselves here to a certain schematic model that is inspired by, but not necessarily mathematically equivalent to, the microscopic equations presented in Ref. 99. Since the model has been successfully used in data analysis, the restriction appears plausible.

The paper is organized as follows: in Sec. III we summarize the equations defining the model. The longtime limits of the correlation functions, characterizing the glassy and localized states, are discussed in Sec. III. Sections [V] and $\mathrm{V}$ are devoted to deriving asymptotic expressions for times large compared to the single-particle relaxation time, valid on intermediate- respectively longtime windows that open upon approaching the transition points. These are the analogues to the common MCT scaling laws referred to as $\beta$ - and $\alpha$-scaling. In Sec. VI we transfer these results to a two-parameter scaling law for the friction coefficient, after which Sec.VII concludes.

\section{SCHEMATIC MODE-COUPLING THEORY}

We summarize the main equations defining the schematic MCT model for active nonlinear microrheology. Following a generic integration-through-transients (ITT) scheme and the notion that the slow dynamics in the vicinity of the (colloidal) glass transition is dominated by density fluctuations [19, the central quantities of the model are the transient density correlation functions. While in the liquid state, these eventually decay to zero, in the glass they attain a finite positive long-time limit called the glass form factor or nonergodicity parameter $f$. Even in the liquid, the correlation functions stay close to its value $f_{c}$ at the transition over an increasingly large time window as one approaches the transition. The quantity $f$ and its tagged-particle counterpart $f^{s}$ will play a central role in the discussion to follow.

In the specific model we choose, there is one correlator $\phi(t)$ mimicking the dynamics of the host liquid and determining the glass form factor $f=\lim _{t \rightarrow \infty} \phi(t)$. Furthermore, this dynamics is taken as identical to the equilibrium one. Clearly, in the thermodynamic limit and assuming that the probe-host interactions remain sufficiently short-ranged, the host-liquid dynamics is in the ensemble average unperturbed by the external force that is applied to the probe particle only. The macroscopic state of the host (liquid or glassy) will then be determined by the equilibrium coupling coefficients only. The equation of motion for the host-liquid correlator takes 
the form

$$
\partial_{t} \phi(t)+\Gamma\left\{\phi(t)+\int_{0}^{t} m\left(t-t^{\prime}\right) \partial_{t^{\prime}} \phi\left(t^{\prime}\right) d t^{\prime}\right\}=0 .
$$

Equations of this type can be derived from microscopic starting points using a Mori-Zwanzig projection operator scheme [15], where the slow relaxation is modeled by a memory kernel $m(t)$ determined by the fluctuating forces. The coefficient $\Gamma$ is a relaxation rate of the short-time dynamics, and will be set to unity in the calculations below (thus defining the unit of time). The central idea of MCT is to approximate the memory kernel by a bilinear form of the correlators themselves, expressing the notion that a slow decay of density fluctuations leads to and hinges upon slow decorrelation of fluctuating forces. In our discussion we adopt the so-called $\mathrm{F}_{12}$ model,

$$
m(t)=v_{1} \phi(t)+v_{2} \phi(t)^{2},
$$

with positive parameters $\left(v_{1}, v_{2}\right)$. With this choice, the full range of asymptotic behavior expected from the microscopic MCT close to ordinary liquid-glass transitions is reproduced [15]. The use of one single mode to describe the host dynamics embodies our assumption that the system remains homogeneous and isotropic in the ensemble average.

Spatial isotropy clearly breaks down for the motion of the probe particle. Therefore, in the schematic model of Ref. [10], two tagged-particle correlation functions $\phi_{\alpha}^{s}(t)$ were introduced, labeled by $\alpha \in\{\|, \perp\}$ to indicate the separate role played by fluctuations in direction of, and perpendicular to, the applied force. The equations of motion then read

$$
\partial_{t} \phi_{\alpha}^{s}(t)+\omega_{\alpha}\left\{\phi_{\alpha}^{s}(t)+\int_{0}^{t} m_{\alpha}^{s}\left(t-t^{\prime}\right) \partial_{t^{\prime}} \phi_{\alpha}^{s}\left(t^{\prime}\right) d t^{\prime}\right\}=0 .
$$

Here, the initial relaxation rates are $\omega_{\perp}=\Gamma_{s}$ and $\omega_{\|}=$ $\Gamma_{s}\left(1-i \kappa_{\|} F_{\mathrm{ex}}\right)$, which fixes the unit of energy as $k_{\mathrm{B}} T=1$. We furthermore set $\Gamma_{s}=1$ for simplicity, suitable for a probe that is almost identical to the host particles. $\kappa_{\|}$ adjusts the scale of forces entering the schematic model. The slow dynamics of the probe arises as a consequence of slow dynamics in the host liquid, and is modeled by memory kernels containing a linear coupling to $\phi(t)$,

$$
\begin{aligned}
m_{\|}^{s}(t) & =\left(v_{1}^{s} \phi_{\|}^{s *}(t)+v_{2}^{s} \phi_{\perp}^{s}(t)\right) \phi(t) /\left(1-i \kappa_{\|} F_{\mathrm{ex}}\right), \quad(3 \mathrm{~b}) \\
m_{\perp}^{s}(t) & =\left(v_{1}^{s} \phi_{\perp}^{s}(t)+v_{2}^{s} \operatorname{Re} \phi_{\|}^{s}(t)\right) \phi(t) /\left(1+\left(\kappa_{\perp} F_{\mathrm{ex}}\right)^{2}\right) .
\end{aligned}
$$

The specific choice of terms entering Eqs. (3) is rooted in symmetry considerations based on the full microscopic MCT model [9, 10]. Specifically, for $F_{\mathrm{ex}}=0$, the model reduces to a well-studied schematic model of taggedparticle motion close to the glass transition, the Sjögren model 20]. In this case $\phi_{\|}^{s}(t) \equiv \phi_{\perp}^{s}(t)$, both are realvalued, and only a single coupling strength $v^{s}=v_{1}^{s}+v_{2}^{s}$ is relevant. Assuming that the probe motion retains rotational symmetry around the axis set by $F_{\text {ex }}$ in the ensemble average, it is seen that $\phi_{\perp}^{s}(t)$ remains a real-valued function. On the other hand, a nonvanishing net displacement of the probe along the direction of the force is expected, which results in the modulation of the corresponding Fourier-transformed density-fluctuation correlations with a complex phase. Hence, $\phi_{\|}^{s}(t)$ will be complex-valued. Furthermore, Eqs. (3) obey the expected symmetries under reversal of the applied force, $F_{\text {ex }} \mapsto-F_{\text {ex }}$. The parameters $\kappa_{\|}$and $\kappa_{\perp}$ characterize the forces relevant for inducing the decay of fluctuations in the two directions. They are introduced to achieve quantitative fits of simulation and experimental data [10. We fix $\kappa_{\perp}=1 / 2$ and $\kappa_{\|}=1$ for the numerical calculations presented below.

We are thus left with a three-correlator model where four parameters enter that are relevant for an understanding of the qualitative long-time behavior: two of them, $\left(v_{1}, v_{2}\right)$ model the approach to the glass transition in the host liquid. In the parameter space of the $\mathrm{F}_{12}$ model, a line of ideal glass transitions $\left(v_{1, c}, v_{2, c}\right)$ exists, and it is useful to characterize the distance to any chosen point on this line by a single distance parameter $\varepsilon$. For the discussion below, let us fix $v_{2, c}=2$ implying $v_{1, c}=2(\sqrt{2}-1)$ [15], and set $\left(v_{1}, v_{2}\right)=\left(v_{1, c}, v_{2, c}\right)(1+\varepsilon)$. As usual, $\varepsilon<0$ signals liquid states, while $\varepsilon>0$ holds for glassy states. We will denote quantities calculated at glass-transition points of the host-liquid model by subscripts $c$.

The other two parameters, $\left(v_{1}^{s}, v_{2}^{s}\right)$, represent the coupling of the probe to the host liquid; they will, among other things, also reflect a non-trivial size ratio between probe and host particles. We employ the simplification that was used in Ref. [10] and set $v_{1}^{s} / v_{2}^{s}=2$ for numerical calculations. Note that setting $v_{2}^{s}=0$ in Eqs. (3) reduces the model to the one originally proposed in Ref. 9, not taking into account the role of probe-density fluctuations perpendicular to the force direction.

While the transient correlation functions can in principle be measured and have been evaluated in computer simulation [9, 10], an experimentally more easily accessible quantity is the friction coefficient $\zeta$. A straightforward adaption of the microscopic expression, used in [10, is $\zeta=1+\Delta \zeta$ with

$$
\begin{aligned}
\Delta \zeta=\mu \int_{0}^{\infty} \phi(t) \phi_{\perp}^{s}(t) d t & \\
& +(1-\mu) \int_{0}^{\infty} \phi(t) \operatorname{Re} \phi_{\|}^{s}(t) d t .
\end{aligned}
$$

This uses that in our choice of units, the solvent friction experienced by the free particle is unity, $\zeta=1$. In the microscopic theory, $\Delta \zeta$ is given as an angular average over a force-autocorrelation function; approximating the latter in terms of density-pair modes, one gets a Fourier-space integral over anisotropic coupling coefficients. In writing Eq. (4), we assume that this integral is qualitatively dom- 


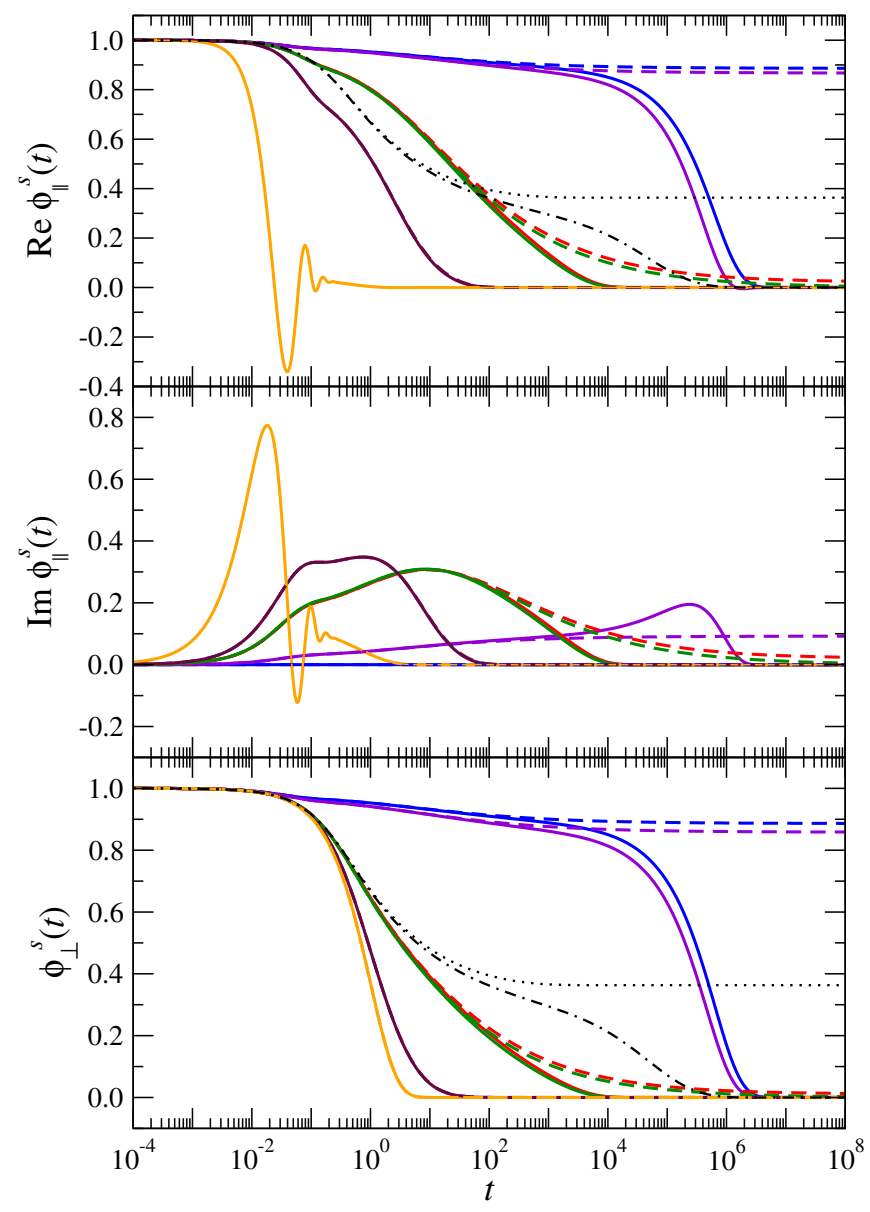

FIG. 1. Schematic-model correlation functions $\phi_{\|}^{s}(t)$ and $\phi_{\perp}^{s}(t)$ for host-liquid parameters close to and at the glass transition, $\varepsilon=-10^{-2}$ (solid lines) and $\varepsilon=0$ (dashed), for probe-coupling coefficients $v^{s}=30, \kappa_{\perp}=0.5$, and $\kappa_{\|}=1$. For $\phi_{\|}^{s}(t)$, both real and imaginary part are shown in separate panels. Curves in the order of decreasing relaxation time correspond to $F_{\mathrm{ex}}=0,1, F_{\mathrm{ex}}^{\mathrm{c}}-0.1, F_{\mathrm{ex}}^{\mathrm{c}}, 12$, and 80 , where $F_{\mathrm{ex}}^{\mathrm{c}}=6.5735$. In the panels showing the real parts, the host-liquid correlation functions $\phi(t)$ are added for the liquid (dash-dotted lines) and glassy (dotted) state.

inated by contributions from the two modes considered in the schematic model, and that in particular the qualitative features of the correlators $\alpha=\|$ and $\alpha=\perp$ are not restricted to zero-measure portions of wave-vector space. The parameter $\mu$ allows to reweight these contributions, which does not qualitatively change the features close to the delocalization transition. Following Ref. [10] we set $\mu=1 / 2$ in our calculations.

Figure 1 displays exemplary correlation functions of the schematic model both in the liquid state (solid lines) and at the liquid-glass transition point (dashed). Numerical solutions of Eqs. (2) to (3) are obtained by integrating in the time domain, using a repeated doubling of the integration step to allow covering a large number of decades in time. The algorithm is a straightforward generalization of the one used in previous MCT calculations
[29.

Considering first $F_{\mathrm{ex}}=0$, the correlation functions are all real, and Fig. 1 demonstrates the two-step decay typical for glassy structural relaxation: at times much larger than those associated with single-particle motion, $t \gg 1 / \Gamma$, a window of structural relaxation opens. Correlators first decay towards a finite plateau, identifying by $\phi_{\alpha}^{s}\left(t \approx t_{\sigma}\right) \approx f_{\alpha}^{s}$ the so-called $\beta$-relaxation regime. The time scale $t_{\sigma}$ diverges approaching the glass transition. In the glass, and right at the glass transition $(\varepsilon=0)$, the correlation functions never decay from their plateau. In the liquid, a final decay to zero sets in on time scales large compared to those of the $\beta$ relaxation, $t \gg t_{\sigma}$. This identifies the $\alpha$-relaxation window $t / t_{\sigma}^{\prime}=\mathcal{O}(1)$ and a second time scale $t_{\sigma}^{\prime}$ that diverges faster than $t_{\sigma}$ upon approaching the glass transition. The equilibrium tagged-particle correlators inherit these properties from the host-liquid correlator $\phi(t)$, shown in Fig. 1 as dash-dotted (liquid) and dotted (glass) lines. Linear response is the regime for small $F_{\text {ex }}$ where the $\phi_{\alpha}^{s}(t)$ are still close to their $F_{\text {ex }}=0$ limiting cases, as in this case, $\zeta$ defined through Eq. (4) remains force-independent.

Increasing $F_{\text {ex }}$ in the schematic model leads to a decrease of the plateau in the $\phi_{\alpha}^{s}(t)$, and to a decrease of their $\alpha$-relaxation time. It can be argued $[9$ that this corresponds to the fact that the localized probability density for the location of the probe particle continuously broadens when increasing $F_{\mathrm{ex}}$. For large enough external forces, the plateau vanishes completely, corresponding to delocalized probe motion. This allows to define a critical or threshold force $F_{\mathrm{ex}}^{\mathrm{c}}$. For glassy states, this indicates the force needed to locally melt the glass surrounding the probe. In the liquid, the nearest-neighbor cages giving rise to glassy dynamics still persist over a time scale $t_{\sigma}^{\prime}$, and around $F_{\text {ex }}^{\mathrm{c}}$ these are broken faster and more effectively by the applied force than by thermal fluctuations. For the parameters used in Fig. 1, the following analysis confirms $F_{\mathrm{ex}}^{\mathrm{c}} \approx 6.5735$.

At still larger forces, the correlator $\phi_{\|}^{s}(t)$ in Fig. 1 shows oscillatory behavior. This can be connected to a finite average probe motion in the delocalized state, as we will investigate in more detail below. For $\phi_{\perp}^{s}(t)$, no such oscillations are seen, as on average the probe will not move perpendicular to the direction of the applied force. The oscillations are a clear signature of the non-equilibrium nature of the dynamics, since for colloidal dynamics, the negative semidefiniteness of the time-evolution operator rules them out in equilibrium [13, 14, 21.

Figure 2 presents curves for the real part of the probeparticle correlation function corresponding to fluctuations in the direction of the force, $\operatorname{Re} \phi_{\|}^{s}(t)$, in a doublelogarithmic plot. Compared to Fig. 11, a state even closer to the glass transition has been taken, in order to bring out more clearly the different time scales and the associated relaxation laws for the correlators. To explain these is the aim of the discussion pursued in this paper. An interesting feature brought out by the analysis pre- 


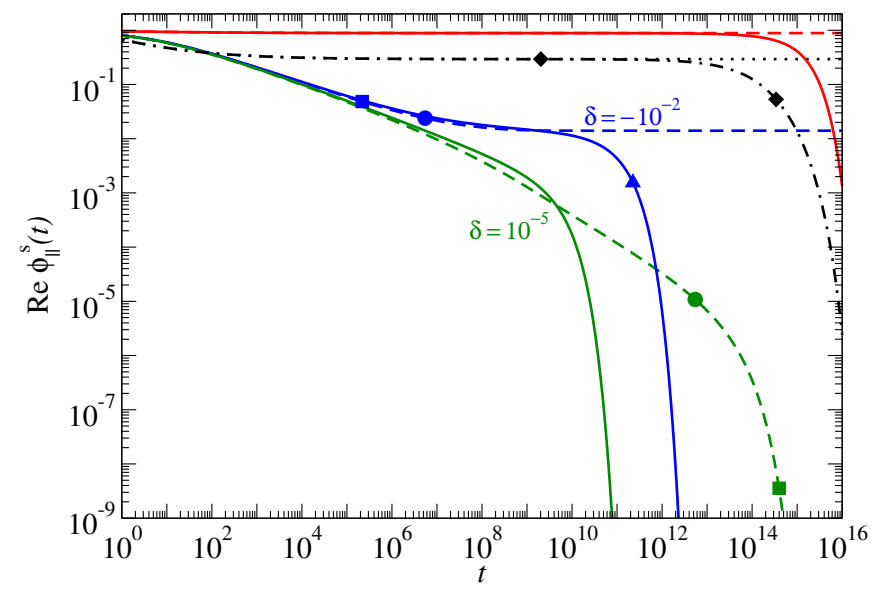

FIG. 2. Real part of the probe-particle correlation functions $\operatorname{Re} \phi_{\|}^{s}(t)$ for the schematic model for force-driven microrheology, for a distance to the host-liquid glass transition $\varepsilon= \pm 10^{-6}$, and forces $F_{\mathrm{ex}}=F_{\mathrm{ex}}^{\mathrm{c}}(1+\delta)$ with $F_{\mathrm{ex}}^{\mathrm{c}} \approx 6.587$ and $\delta=-1,-0.01$, and $10^{-5}$, from top to bottom. Model parameters are $v^{s}=30, \kappa_{\perp}=0.5$, and $\kappa_{\|}=1$ (others as mentioned in the text). Solid (dashed) lines show the results for the liquid (glass). Dash-dotted (dotted) lines show the corresponding host-liquid correlator $\phi(t)$ for the liquid (glass). Symbols mark the time scales $t_{\sigma}$ and $t_{\sigma}^{\prime}$ for the host correlator (diamonds, cf. Eq. (14)), $t_{\delta}$ (squares), $t_{1 / 2}$ (circles), and $t_{\sigma, \delta}^{\prime}$ (triangles) for the probe correlator (Eqs. 29), (30), and (32).

sented below is the fact that all the probe-particle correlation functions are, asymptotically close to the glassand the delocalization transition, proportional to each other. Hence, a discussion of the time scales seen in $\operatorname{Re} \phi_{\|}^{s}(t)$ suffices. They are marked in Fig. 2 by various symbols, and we will come back to their discussion below.

The behavior of the correlation functions shown in Figs. 1 and 2 gives rise to a strongly nonlinear signature in the friction coefficient. Figure 3 shows the friction increment $\Delta \zeta$ calculated by Eq. (4) as a function of external force $F_{\text {ex }}$ for several values of $\varepsilon$; other parameters were chosen as in Fig. 1. Qualitatively, the resulting $\zeta\left(F_{\text {ex }}\right)$ agree with those discussed in Refs. 9, 10] in conjunction with experimental and simulation data. For $F_{\mathrm{ex}} \rightarrow 0$, a linear-response regime is recovered where $\zeta$ depends only weakly on $F_{\mathrm{ex}} \cdot \quad \zeta\left(F_{\mathrm{ex}} \rightarrow 0\right)$ increases strongly with decreasing $|\varepsilon|$ from the liquid side. This is the manifestation of the glass transition, where the equilibrium mobility of the tracer particle vanishes, as long as the coupling between probe and host liquid is sufficiently strong. The possibility of a decoupling of tracer motion from the host even in equilibrium (realized, e.g., by tracers of sufficiently small size in binary mixtures of hard spheres [22]) will not be discussed in this paper.

As $F_{\text {ex }}$ is increased, deviations from linear response set in quadratically with the force, $\Delta \zeta \approx \Delta \zeta\left(F_{\mathrm{ex}} \rightarrow 0\right)-$ $a F_{\mathrm{ex}}^{2}$, with a prefactor $a$ depending on $\varepsilon$. A steep descent of $\Delta \zeta$ is then seen in Fig. 3 around the threshold force,

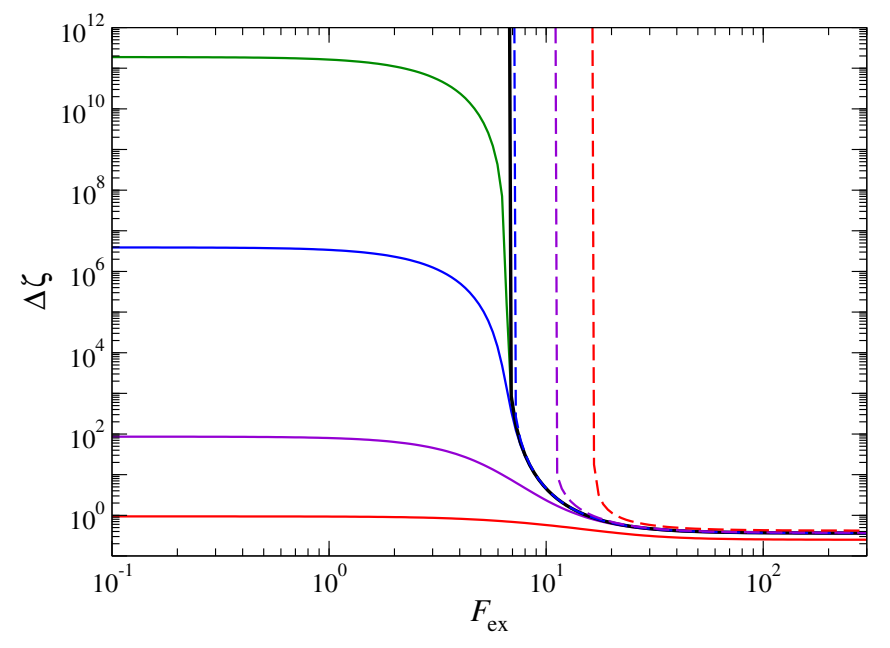

FIG. 3. Excess friction $\Delta \zeta$ experienced by a probe particle, as a function of the applied force $F_{\text {ex }}$, calculated in the schematic MCT model according to Eq. (4). Curves from bottom to top correspond to distances to the host-liquid glass transition $\varepsilon=-1,-0.1,-10^{-3},-10^{-5}$ (liquid; solid lines), 0 (solid), $10^{-3}, 0.1$, and 1 (glass; dashed lines). Other parameters are chosen as in Fig. 1 .

$F_{\mathrm{ex}} \approx F_{\mathrm{ex}}^{\mathrm{c}}$. In the glass, the point $F_{\mathrm{ex}}=F_{\mathrm{ex}}^{\mathrm{c}}$ marks the divergence of $\Delta \zeta$ as one approaches the critical force from above, $F_{\mathrm{ex}} \rightarrow F_{\mathrm{ex}}^{\mathrm{c}}+0$. At forces much larger than $F_{\mathrm{ex}}^{\mathrm{c}}$, a second plateau is observed in $\Delta \zeta$. Intuitive reasoning might suggest $\zeta\left(F_{\text {ex }} \rightarrow \infty\right) \rightarrow 1$ (the solvent friction in our units), hence $\Delta \zeta=0$ in this window. This is not seen in experiment or simulation. It was argued in Ref. 10 that within ITT-MCT, the fact that $\Delta \zeta>0$ for $F_{\mathrm{ex}} \rightarrow \infty$ can be understood by accounting for contributions to $\Delta \zeta$ stemming from $\alpha=\perp$, i.e., fluctuations in the direction perpendicular to the force. Note that no reference to a suspending liquid is made in this argument, although hydrodynamic interactions mediated through the solvent are expected to greatly influence the friction coefficient for large forces in real colloidal suspensions. The ratio of the large-force plateau to the linear-response value has been determined in a low-density expansion by Brady and coworkers [23, 24] to be $\Delta \zeta\left(F_{\mathrm{ex}} \rightarrow \infty\right) / \Delta \zeta\left(F_{\mathrm{ex}} \rightarrow\right.$ $0)=2$; this is confirmed by simulations of dilute host liquids. In the schematic model, the parameter $\mu$ serves to reproduce this ratio when one assumes the coupling coefficients $\left(v_{1}, v_{2}\right)$ and $\left(v_{1}^{s}, v_{2}^{s}\right)$ to approach zero at low densities: dropping all memory kernels from Eqs. (2) and (3), the correlation functions become $\phi(t)=\phi_{\perp}(t)=$ $\exp (-t)$ and $\operatorname{Re} \phi_{\|}(t)=\exp (-t) \cos \left(F_{\text {ex }} t\right)$. In Eq. (4) this yields $\Delta \zeta_{0}=\left(4+\mu F_{\text {ex }}^{2}\right) /\left(8+2 F_{\text {ex }}^{2}\right)$, which interpolates between $\Delta \zeta_{0}\left(F_{\text {ex }} \rightarrow 0\right)=1 / 2$ and $\Delta \zeta_{0}\left(F_{\text {ex }} \rightarrow \infty\right)=\mu / 2$. In Fig. 3 this is not seen, since we kept $v^{s}=30$ fixed. While fits to data and the microscopic theory suggest to change $v^{s}$ as a function of density [10, for a discussion of the features close to the glass transition density we assume $v^{s} \approx v^{s}(\varepsilon=0)$ for simplicity.

We now focus on the analytic discussion of the above 
observed crossovers. For this purpose, let us employ a more compact notation of Eq. (3). We introduce a vector of probe correlation functions, $\phi^{s}(t)$, with components $\left(\phi_{1}^{s}(t), \phi_{2}^{s}(t), \phi_{3}^{s}(t)\right)^{\top}:=\left(\operatorname{Re} \phi_{\|}^{s}(t), \operatorname{Im} \phi_{\|}^{s}(t), \phi_{\perp}^{s}(t)\right)^{\top}$, and initial conditions $\underline{\phi}_{0}^{s}=(1,0,1)^{\top}$. We will refer to the components of this vector with Latin indices $j \in\{1,2,3\}$ (recall that Greek indices, $\alpha \in\{\|, \perp\}$, label the directions). Let us further introduce a matrix characterizing the short-time motion,

$$
\underline{\underline{\omega}}:=\left(\begin{array}{ccc}
1 & \kappa_{\|} F_{\mathrm{ex}} & 0 \\
-\kappa_{\|} F_{\mathrm{ex}} & 1 & 0 \\
0 & 0 & 1
\end{array}\right)
$$

and a matrix constructing the memory kernel vector,

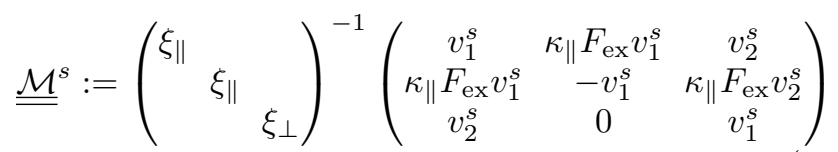

with $1 / \xi_{\alpha}=1+\left(\kappa_{\alpha} F_{\text {ex }}\right)^{2}$. The complex-number multiplication appearing in the convolution integral is then represented in our matrix notation by a bilinear (but nonsymmetric) mapping $\underline{\mathcal{C}}_{j}^{s}[\underline{x}, \underline{y}]:=\underline{x}^{\top} \cdot \underline{\underline{\mathcal{M}}}^{{ }^{\top}} \underline{\underline{C}}_{j} \cdot \underline{y}$, with

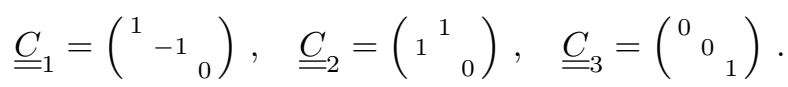

It will occasionally be useful to introduce the symmetrized version of this mapping, $\underline{\mathcal{D}}^{s}[\underline{x}, \underline{y}]=\underline{\mathcal{C}}^{s}[\underline{x}, \underline{y}]+$ $\underline{\mathcal{C}}^{s}[\underline{y}, \underline{x}]$. Applying the Laplace transform, $\hat{\phi}(z)=$ $i \int_{0}^{\bar{\infty}} e^{i z t} \phi(t) d t$, the equations of motion Eqs. (2a) and 3a are rewritten as

$$
z \hat{\phi}(z)-(1+z \hat{\phi}(z))(i z+z \hat{m}(z))=0
$$

for the bath correlator, and

$$
\begin{aligned}
-i z \underline{\underline{\omega}}^{-1}(z \underline{\hat{\phi}} & \left.(z)+\underline{\phi}_{0}^{s}\right)+z \underline{\hat{\phi}}^{s}(z) \\
& -z \underline{\mathcal{C}}^{s}\left[\widehat{\phi \phi^{s}}(z), z \underline{\phi}^{s}(z)+\underline{\phi}_{0}^{s}\right]=0
\end{aligned}
$$

for the probe correlator.

\section{LONG-TIME BEHAVIOR}

We assume the long-time limits of all correlation functions of the schematic model to exist. In view of standard features of structural relaxation dynamics close to a glass transition, this is not unreasonable. Then, the Abelian theorem [25],

$$
\begin{aligned}
f & :=\lim _{t \rightarrow \infty} \phi(t)=-\lim _{z \rightarrow+i 0} z \hat{\phi}(z), \\
\underline{f}^{s} & :=\lim _{t \rightarrow \infty} \underline{\phi}^{s}(t)=-\lim _{z \rightarrow+i 0} z \hat{\phi}^{s}(z),
\end{aligned}
$$

leads from Eqs. (6) to

$$
f /(1-f)=v_{1} f+v_{2} f^{2}
$$

for the bath correlator and

$$
0=\underline{\underline{A}}^{s} \cdot \underline{f}^{s}-f \underline{\mathcal{C}}^{s}\left[\underline{f}^{s}, \underline{f}^{s}\right],
$$

for the probe correlators. Here we have defined

$$
\underline{\underline{A}}^{s} \cdot \underline{f}^{s}:=f \underline{\mathcal{C}}^{s}\left[\underline{f}^{s}, \underline{\phi}_{0}^{s}\right]-\underline{f}^{s} .
$$

Explicit evaluation leads to

$$
\underline{\underline{A}}^{s}=\left(\begin{array}{ccc}
\xi_{\|} v_{1}^{s} f-1 & \xi_{\|} \kappa_{\|} F_{\mathrm{ex}} v_{1}^{s} f & \xi_{\|} v_{2}^{s} f \\
\xi_{\|} \kappa_{\|} F_{\mathrm{ex}} v_{1}^{s} f & -\xi_{\|} v_{1}^{s f}-1 & \xi_{\|} \kappa_{\|} F_{\mathrm{ex}} v_{2}^{s} f \\
\xi_{\perp} v_{2}^{s} f & 0 & \xi_{\perp} v_{1}^{s} f-1
\end{array}\right) .
$$

Equation (8) describes the well-known bifurcation scenario of the glass transition within MCT [15]: it is an implicit nonlinear equation for the nonergodicity parameter $f$. Possibly many different solutions of this equation exist for general models, with zero being an obvious one. It can be proven [13] that within equilibrium MCT, $f$ is always determined by the largest positive real solution. Since for $v_{1,2} \rightarrow 0$, only $f=0$ survives, and for $v_{1,2} \rightarrow \infty, f>0$ holds, the MCT solutions changes at some bifurcation point. These points define a hypersurface $\left(v_{1, c}, v_{2, c}\right)$ through $v_{1, c}=2 \sqrt{v_{2, c}}-v_{2, c}$ (restricting to $\left.1<v_{2, c} \leq 4\right)$. The bifurcation is identified as the ideal glass transition. Generically, in Eq. (8), a jump in $f$ occurs; this is called a type B transition in the literature. In other words, $f_{c}>0$ holds for the critical nonergodicity parameter evaluated right at the transition. Mathematically, one deals for generic mode-coupling models with bifurcations of the class $\mathcal{A}_{\ell}$ [13] according to the Arnol'd classification [26]; in the present case we are only concerned with the nondegenerate $\mathcal{A}_{2}$ bifurcations displayed by the $\mathrm{F}_{12}$ model. The bifurcation points are identified by recognizing that, as two branches of solutions of the implicit equation for $f$ coalesce, the equation is no longer invertible (and the implicit-function theorem violated).

Equation (9) describes localization respectively delocalization of probe particles: if $f=0$, also $f^{s}=0$, i.e. in a liquid, all probe particles are delocalized (and able to undergo long-range motion, as required for a liquid). If $f>0$, sufficiently large $\left(v_{1}^{s}, v_{2}^{s}\right)$ will for $F_{\mathrm{ex}}=0$ lead to $f_{\alpha}^{s}>0$, so that a probe is localized in the glass if it couples strongly enough. As noted above, we will not discuss the weak-coupling limit where the probe remains delocalized in the glass even for $F_{\mathrm{ex}}=0$.

For non-zero $F_{\text {ex }}$, a further bifurcation point will describe probe delocalization in the glass through external force. It can be found by demanding that the implicitfunction theorem is violated for Eq. (9), $\operatorname{det} \underline{\underline{A}}^{s}=0$, leading to a biquadratic equation,

$$
\begin{array}{r}
0=\kappa_{\perp}^{2} \kappa_{\|}^{2}\left(F_{\mathrm{ex}}^{\mathrm{c}}\right)^{4}-\left(v_{1}^{s} f-1\right)\left(\kappa_{\perp}^{2}\left(v_{1}^{s} f+1\right)+\kappa_{\|}^{2}\right)\left(F_{\mathrm{ex}}^{\mathrm{c}}\right)^{2} \\
+\left(v_{1}^{s} f+1\right)\left(\left(v_{1}^{s} f-1\right)^{2}-\left(v_{2}^{s} f\right)^{2}\right) \cdot
\end{array}
$$



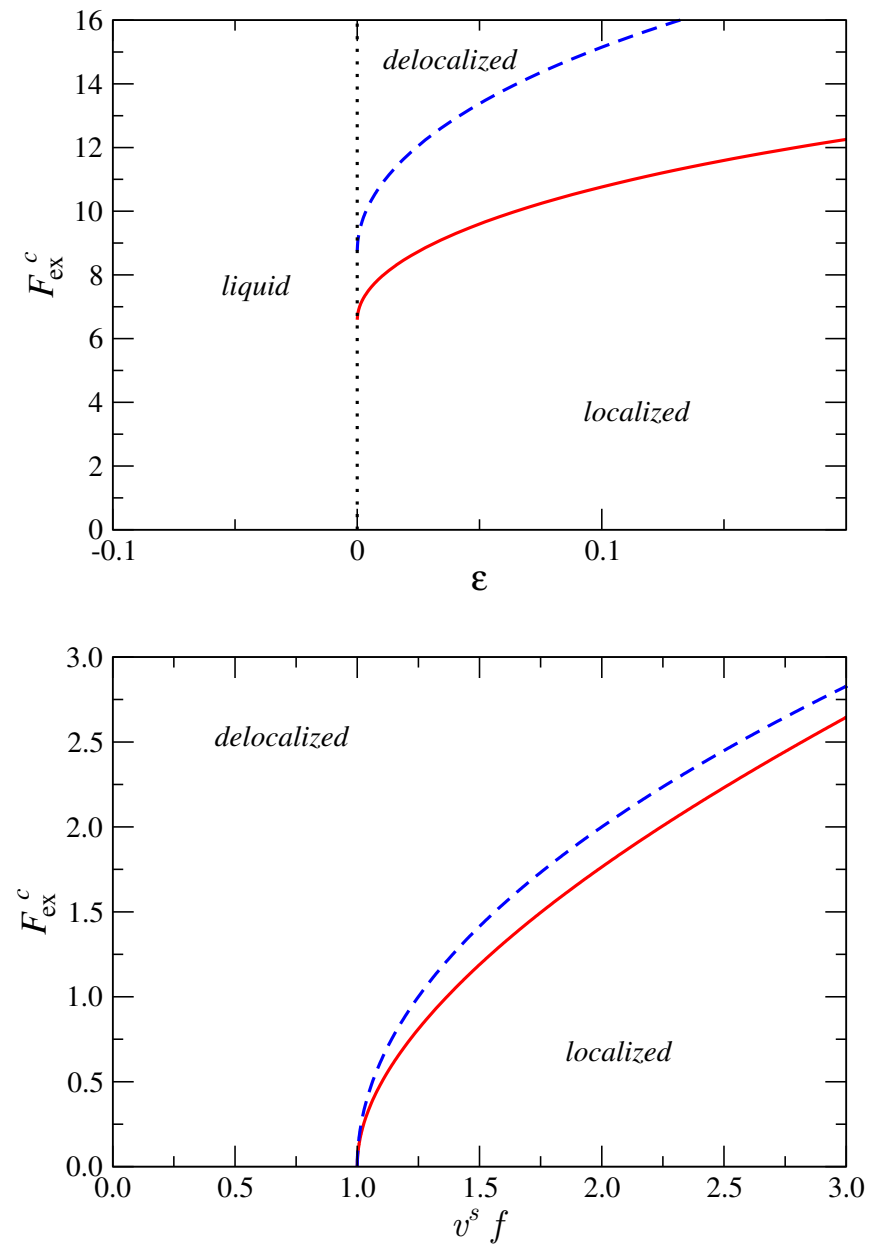

FIG. 4. State diagram for the probe particle in the schematic model. Upper panel: critical force $F_{\mathrm{ex}}^{\mathrm{c}}$ as a function of distance to the host-liquid glass transition $\varepsilon$, for fixed $v^{s}$ as in Fig. 1. Lower panel: critical force as a function of probeto-host coupling strength $v^{s} f$. Solid lines mark the boundary between localized and delocalized probe states; a vertical dotted line in the upper panel indicates the host-liquid glass transition. Dashed lines are $F_{\mathrm{ex}}^{\mathrm{c}}$ obtained for the model without taking into account the anisotropy of the probe-particle fluctuations, $v_{2}^{s}=0$.

Solving Eq. 11) to obtain $F_{\mathrm{ex}}^{\mathrm{c}}$ as a function of $v^{s} f$, we obtain a decomposition of the parameter space for our schematic model. The result for exemplary parameters is shown in Fig. 4. The lower panel shows $F_{\mathrm{ex}}^{\mathrm{c}}$ as a function of $v^{s} f$ directly. Typically, $v^{s} f$ will change implicitly as the glass form factor $f$ changes with changing $\varepsilon$; this variation is shown in the upper panel of the figure for the parameters chosen as above. In Fig. 4, also the result after setting $v_{2}^{s}=0$ (as implicit in the model of Ref. 9]) is shown for comparison (dashed lines). For this aspect of the discussion, both models are very similar. For $v^{s} f<1$, no real solution of Eq. (11) exists, and the probe particle is always delocalized. This is trivially the case in the liquid, where $f=0$. In the glass, $f>0$ holds, and by assumption we restrict ourselves

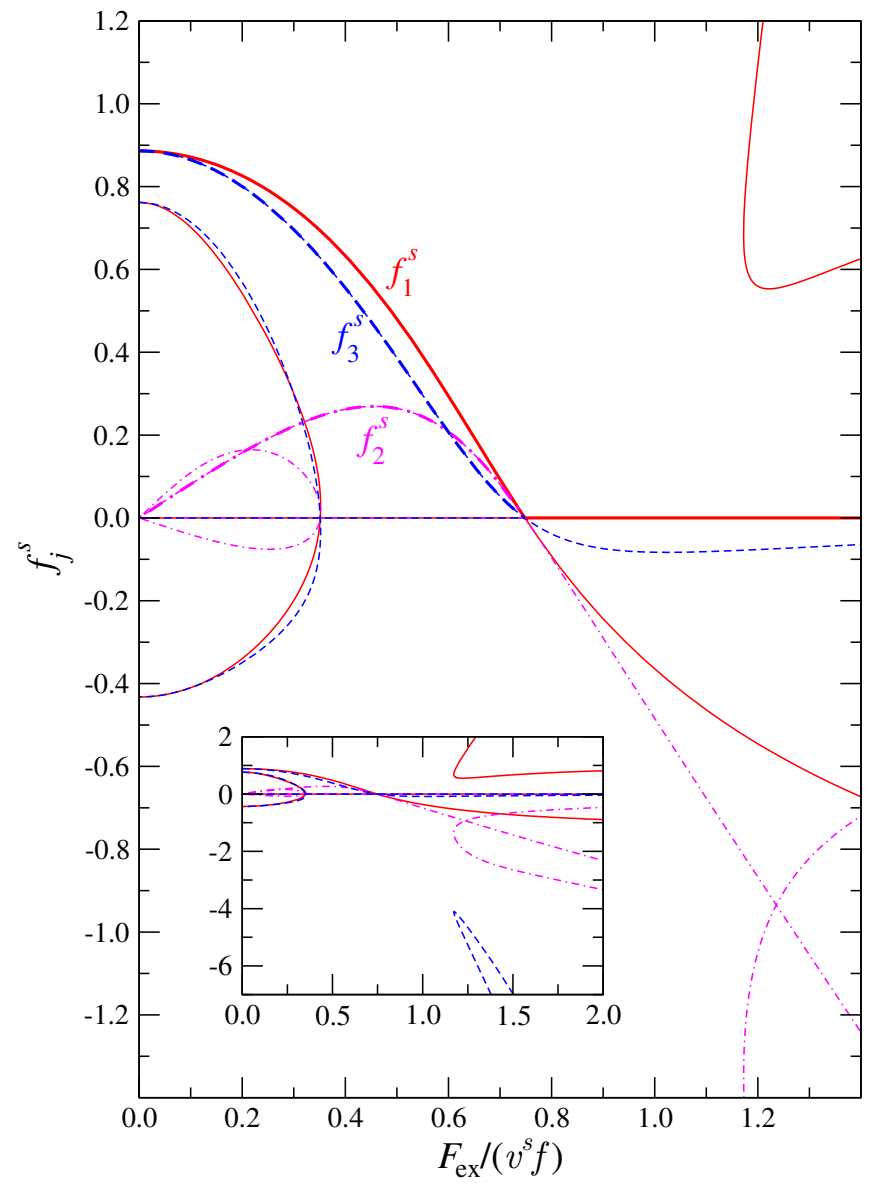

FIG. 5. Solutions of the implicit set of equations Eq. 9, $f_{1}^{s}=\operatorname{Re} f_{\|}^{s}$ (solid lines), $f_{2}^{s}=\operatorname{Im} f_{\|}^{s}$ (dash-dotted), and $f_{3}^{s}=f_{\perp}^{s}$ (dashed), as a function of the reduced applied force, $F_{\text {ex }} /\left(v^{s} f\right)$. Parameters are $v^{s}=30, f=1-1 / \sqrt{2}$ corresponding to the glass-transition point of the $\mathrm{F}_{12}$ model at $v_{2}^{c}=2$. The solutions identified as the long-time limits of the schematic model, Eq. (3), are shown as thick lines. The inset shows the full set of solution branches, including one for $f_{3}^{s}$ that is cut off in the full figure for clarity.

to the case where the probe particle also becomes localized without any external force, so that the jump from zero to $f_{c}$ is big enough to render $v^{s} f_{c}>1$ at the glass transition. As exemplified in the upper panel of Fig. 4, this leads to a nonvanishing critical force $F_{\mathrm{ex}}^{\mathrm{c}}>0$ for the delocalization of the probe at the glass transition, identifying the regime $F_{\mathrm{ex}}<F_{\mathrm{ex}}^{\mathrm{c}}$ as the one where the probe remains localized inside the glass even under the action of an external force. Beyond that, $f$ increases with $\varepsilon$, asymptotically as $f-f_{c} \sim \sqrt{\varepsilon}$. Thus also $F_{\mathrm{ex}}^{\mathrm{c}}$ increases in the glass. A full analysis of Eq. (11) shows that a further line of solutions is present inside this localized regime. Based on the following discussion of $\underline{f}^{s}$, we do not assign physical significance to this.

Solving Eq. (9) numerically, we obtain a set of solutions to this implicit equation. These are shown in Fig. 5 , where we have highlighted those in bold that we will use 
in the following as the physical ones. For $F_{\mathrm{ex}}<F_{\mathrm{ex}}^{\mathrm{c}}$ they are the continuation of the known solution $f_{\alpha}^{s}\left(F_{\mathrm{ex}}=0\right)$. In particular, $f_{2}^{s} \rightarrow 0$ as $F_{\mathrm{ex}} \rightarrow 0$ since the imaginary part of the correlation function has to vanish in equilibrium, and $f_{1}^{s}=f_{3}^{s}$ for $F_{\text {ex }}=0$. The physical branch crosses zero at $F_{\mathrm{ex}}^{\mathrm{c}}$, and for larger $F_{\mathrm{ex}}$, no solution emerges for which both real parts, $f_{1}^{s}$ and $f_{3}^{s}$, are non-negative real, except $f_{\alpha}^{s} \equiv 0$. We hence are dealing with a continuous probe-delocalization transition: the probe-correlator nonergodicity parameters $f^{s}$ do not exhibit a jump at $F_{\mathrm{ex}}^{\mathrm{c}}$. Note that the choice of solution branches is nontrivial: in standard equilibrium MCT, it can be proven that the solution that is the largest nonnegative for all components has to be chosen [13. The proof relies on mathematical properties of Eq. $\sqrt{8}$ and its proper generalizations, that are not ensured in general in Eq. (9). The number of solution branches to Eq. (9) may depend on the parameters, and in particular the choice of the $v_{1}^{s}$ and $v_{2}^{s}$. Fig. 5 shows a case that is typical for the description of microrheology data.

It can be seen that at $F_{\mathrm{ex}}=F_{\mathrm{ex}}^{\mathrm{c}}$, the largest real eigenvalue of the matrix $\underline{\underline{A}}^{s}$ crosses zero, and that this eigenvalue is always non-degenerate for the restriction to the parameters introduced above. Thus the critical force is determined by a bifurcation in Eq. (9) with a single critical direction (co-dimension $d_{c}=1$ ). It is in this sense similar to the known continuous delocalization transitions discussed within tagged-particle models of MCT [15. These are quite different from the standard MCT bifurcation, as we are not dealing with the coalescence of two solution branches, but with the crossing of one particular solution with the $f_{j}^{s}=0$ branch. $F_{\mathrm{ex}}^{\mathrm{c}}$ is welldefined since setting any of the $f_{j}^{s}=0$ in Eq. (9) also demands that the other $f_{i \neq j}^{s}=0$.

For the later discussion we define reduced distances to the critical points of the model, $\varepsilon$ and $\delta$. We set

$$
\begin{gathered}
\underline{V}=\left(v_{1}, v_{2}\right)^{\top}=\underline{V}_{c}+\varepsilon \cdot \underline{b}_{c}, \\
\underline{W}=\left(v_{1}^{s}, v_{2}^{s}, F_{\mathrm{ex}}\right)^{\top}=\underline{W}^{c}+\delta \cdot \underline{b}^{s, c} .
\end{gathered}
$$

The vectors $\underline{b}_{c}$ and $\underline{b}^{s, c}$ can in prinicple be chosen arbitrarily. In particular, the weak-coupling limit of small $v^{s}$ is contained in the following derivation. For the sake of simplicity, we will choose for all explicit calculations, in agreement with above,

$$
\underline{b}_{c}=\left(v_{1, c}, v_{2, c}\right)^{\top}, \quad \underline{b}^{s, c}=\left(0,0, F_{\mathrm{ex}}^{\mathrm{c}}\right)^{\top} .
$$

With this choice, $\delta$ becomes a reduced force, such that $\delta<0$ corresponds to $F_{\mathrm{ex}}<F_{\mathrm{ex}}^{\mathrm{c}}$, i.e., the localized regime, and $\delta>0$ to the delocalized regime. Recall that subscripts $c$ refer to quantities evaluated at $\varepsilon=0$; we reserve superscripts $c$ for quantities evaluated at $\delta=0$.

\section{THE BETA-SCALING LAW}

\section{A. Scaling Functions}

Having identified the long-time limits of the correlation functions in the glassy-host, localized-probe regime, we now turn to a discussion of the dynamical correlation functions $\phi(t)$ and $\phi_{\alpha}^{s}(t)$. We anticipate that upon approaching a critical point, $\varepsilon \rightarrow 0$ for small $\delta$, the correlators will stay close to an intermediate plateau given by $f$ over a time window that increases as the distance parameters approach zero. Thus, $\phi(t)-\tilde{f}$ and $\phi^{s}(t)-\tilde{f}^{s}$ can be identified as small parameters on a dynamical time scale that will be determined below. Here, $\tilde{f}$ is a parameter introduced in Ref. [27], approximating $f$ for $\varepsilon \geq 0$ and continuing the latter smoothly to $\varepsilon<0$ such that $\lim _{\varepsilon \rightarrow \pm 0} \tilde{f}=f_{c}$. For the $\mathrm{F}_{12}$ model, where we only consider $\mathcal{A}_{2}$ bifurcations, $\tilde{f}$ is given by the unique positive solution of $v_{1}+2 v_{2} \tilde{f}-1 /(1-\tilde{f})^{2}=0$ that obeys $\tilde{f}=f_{c}$ at the critical point. We define $\tilde{\tilde{f}}^{s}$ and a corresponding matrix $\tilde{A}^{s}$ by Eqs. (9) and (10) with $f$ replaced by $\tilde{f}$.

For the host correlator $\phi$ given by Eqs. (2), the asymptotic analysis has been worked out in detail. Splitting $\phi(t)=\tilde{f}+G(t)$, there holds an expansion in terms of the small parameter $\sigma: G(t)=c_{\sigma} h g_{\sigma}(t)+\mathcal{O}\left(c_{\sigma}^{2}\right)$ with $c_{\sigma}=\sqrt{|\sigma|}=\mathcal{O}(\sqrt{|\varepsilon|})$. The leading order correction to the plateau is called $\beta$-correlator $g_{\sigma}(t)$. There appears a critical amplitude $h$, connected to the critical eigenvector arising in the bifurcation analysis of Eq. (8) - in the onecorrelator schematic model, this is just a prefactor, set to $h=\left(1-f_{c}\right)$ by convention. The function $g(t)$ describes relaxation to and from the plateau (visible e.g. in Fig. 1) as asymptotic power laws,

$$
\begin{array}{ll}
g_{\sigma}(t) \propto\left(t / t_{0}\right)^{-a} & \text { for } t_{0} \ll t \ll t_{\sigma}, \\
g_{\sigma}(t) \propto-\left(t / t_{\sigma}\right)^{b} & \text { for } t_{\sigma} \ll t \ll t_{\sigma}^{\prime},
\end{array}
$$

where the latter, called the von Schweidler law, only occurs inside the liquid, $\varepsilon<0$. Here, diverging time scales have been identified,

$$
t_{\sigma}=t_{0} /|\sigma|^{1 /(2 a)}, \quad t_{\sigma}^{\prime}=t_{0} /|\sigma|^{1 /(2 a)+1 /(2 b)},
$$

where $\sigma$ is a distance parameter obeying $\sigma \sim \varepsilon$ as $\varepsilon \rightarrow$ 0 . For the schematic model and our definition of $\varepsilon$, one gets $\sigma=\varepsilon f^{c}\left(1+f^{c}\right)\left(1-f^{c}\right) \approx 0.38 \varepsilon$. The microscopic time scale $t_{0}$ is fixed by the short-time motion of the correlation function. The power-law exponents $a, b>0$ are solutions of

$$
\frac{\Gamma(1-a)^{2}}{\Gamma(1-2 a)}=\frac{\Gamma(1+b)^{2}}{\Gamma(1+2 b)}=\lambda,
$$

with the exponent parameter $\lambda$ given by $\lambda=1 / \sqrt{v_{2}}$ on the bifurcation manifold [15].

Introducing a rescaled time, $\hat{t}=t / t_{\sigma}$, the $\beta$ correlator is found to obey the $\beta$-scaling equation written with the scaled Laplace frequency $\hat{z}=z t_{\sigma}$ as

$$
\mp 1+\lambda \hat{z} \widehat{g_{ \pm}^{2}}(\hat{z})+\left(\hat{z} \hat{g}_{ \pm}(\hat{z})\right)^{2}=0
$$


where $g_{ \pm}(\hat{t})$ are the scaling solutions for $\sigma \gtrless 0$ that depend only on the sign of $\sigma$. They obey $g_{ \pm}(\hat{t}) \sim \hat{t}^{-a}$ for $\hat{t} \rightarrow 0$, and $g_{-}(\hat{t}) \sim-B \hat{t}^{b}$ for $\hat{t} \rightarrow \infty$. The asymptotic form of the relaxation to and from the plateau thus is given by a scaling law with only $\lambda$ as a non-universal parameter. The positive constant $B=\mathcal{O}(1)$ depends on $\lambda$ and has been tabulated [28]. For $\varepsilon>0, g_{+}(\hat{t})$ approaches a constant as $\hat{t} \rightarrow \infty$, giving the correction to the critical plateau value.

In the same spirit we now write

$$
\underline{\phi}^{s}(t)=\underline{\tilde{f}}^{s}+c_{\sigma} \underline{g}_{\sigma}^{s}(\hat{t})+\mathcal{O}\left(c_{\sigma}^{2}\right) .
$$

To arrive at an equation for $g_{\sigma}^{s}$ that is asymptotically valid for $\sigma \sim \varepsilon \rightarrow 0$, we follow the standard procedure laid out in Ref. 15: introducing $\hat{z}=z t_{\sigma}$, we collect in Eq. (6b) the leading terms in $c_{\sigma}$, making use of Eq. (9). We arrive at

$$
\begin{aligned}
& 0=\left\{\underline{\underline{\tilde{A}}}^{s} \cdot \hat{z} \underline{\hat{g}}_{\sigma}^{s}(\hat{z})-\tilde{f} \underline{\mathcal{D}}^{s}\left[\underline{\tilde{f}}^{s}, \hat{z} \underline{\hat{g}}_{\sigma}^{s}(\hat{z})\right]\right. \\
& \left.+\hat{z} \hat{g}_{\sigma}(\hat{z}) \underline{\mathcal{C}}^{s}\left[\underline{\tilde{f}}^{s}, \underline{\phi}_{0}^{s}-\underline{\tilde{f}}^{s}\right]\right\} \\
& +c_{\sigma}\left\{\underline{\mathcal{C}}^{s}\left[\hat{z} \widehat{g_{\sigma} \underline{g}_{\sigma}^{s}}(\hat{z}), \underline{\phi}_{0}^{s}-\underline{\tilde{f}}^{s}\right]+\hat{z} \hat{g}_{\sigma}(\hat{z}) \underline{\mathcal{C}}^{s}\left[\underline{\tilde{f}}^{s}, \hat{z} \underline{g}_{\sigma}^{s}(\hat{z})\right]\right. \\
& \left.+\tilde{f} \underline{\mathcal{C}}^{s}\left[\hat{z} \hat{g}_{\sigma}^{s}(\hat{z}), \hat{z} \hat{g}_{\sigma}^{s}(\hat{z})\right]\right\}+\mathcal{O}\left(c_{\sigma}^{2},\left(c_{\sigma} t_{\sigma}\right)^{-1}\right)
\end{aligned}
$$

Note that since the exponent $a<1 / 2$ [15], the dropped terms $\left(c_{\sigma} t_{\sigma}\right)^{-1} \sim|\sigma|^{1 /(2 a)-1 / 2}$ are indeed of higher order.

The leading order for $\varepsilon \rightarrow 0$ in Eq. (18) results in a linear equation system,

$$
\underline{\underline{L}}_{c}^{s} \cdot \underline{g}_{ \pm}^{s}(\hat{t})=g_{ \pm}(\hat{t}) \underline{l}_{c}^{s},
$$

where the linear mapping $\underline{\underline{L}}_{c}^{s}$ is defined through $\underline{\underline{\underline{L}}}_{c}^{s}=$ $\underline{\underline{A}}_{c}^{s}-f_{c} \underline{\mathcal{D}}_{c}^{s}\left[\underline{f}_{c}^{s}, \cdot\right]$ and the inhomogeneity reads $\underline{\underline{\underline{l}}}_{c}^{c}=$ ${ }^{-} \underline{\mathcal{C}}^{s}\left[\underline{f}_{c}^{s}, \underline{\phi}_{0}^{s}-\underline{f}_{c}^{s}\right]$. For state points far enough from the critical force, Eq. 19p determines $\underline{g}_{ \pm}^{s}(\hat{t})$ by the host liquid $g_{ \pm}(\hat{t})$. This expresses that in such cases, the asymptotic dynamics of the tracer is governed by that of the host liquid, i.e.,

$$
\underline{g}_{ \pm}^{s}(\hat{t})=\underline{h}_{c}^{s} g_{ \pm}(\hat{t}), \quad \delta \neq 0,
$$

with an amplitude given by

$$
\underline{h}_{c}^{s}=-\underline{\underline{L}}_{c}^{s-1} \underline{\mathcal{C}}^{s}\left[\underline{f}_{c}^{s}, \underline{\phi}_{0}^{s}-\underline{f}_{c}^{s}\right] .
$$

In Fig. 6, we show exemplary results for the correlators $\phi^{s}(t)$ close to the glass transition, in a doublelogarithmic plot of $\left|\phi^{s}(t)-f^{s}\right|$ to exhibit the asymptotic power laws. Two states corresponding to small $|\varepsilon|$ were chosen that exhibit a large window of validity for the asymptotic Eq. (18). As dash-dotted and dotted lines, the corresponding $\phi(t)$ are shown for $\varepsilon<0$ and $\varepsilon>0$, respectively. They follow the asymptotic critical power law, $\phi(t)-f^{c} \sim h\left(t / t_{0}\right)^{-a}$ over several decades in time; a numerical estimate yields $t_{0} \approx 0.146$ for the parameters

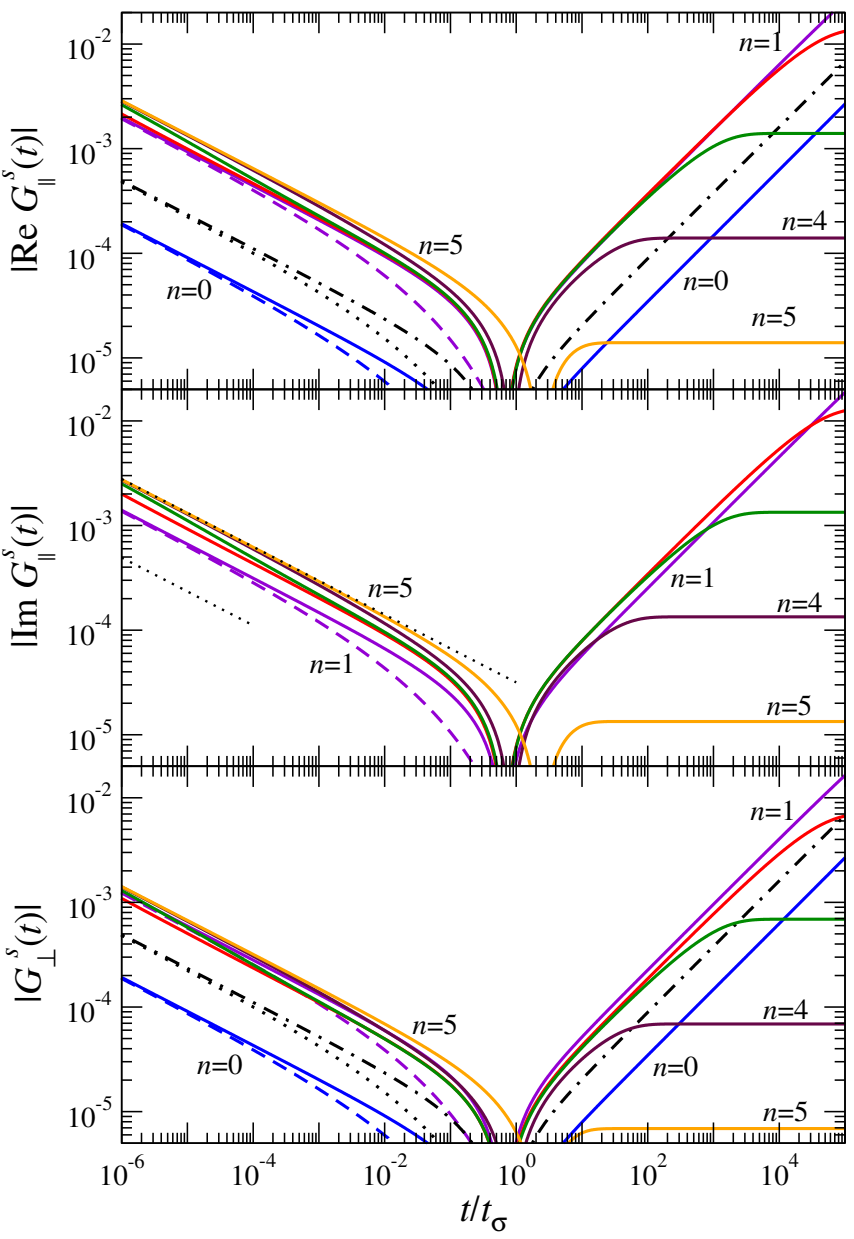

FIG. 6. Correlation functions $\phi^{s}(t)$ close to the glass transition point, for various external forces, plotted as the leadingorder deviation from the plateau value, $\left|\underline{G}^{s}(t)\right|=\left|\phi^{s}(t)-f^{s}\right|$ for $\varepsilon \rightarrow 0$, as a function of $t / t_{\sigma}$. Parameters as in Fig. 3 solid lines correspond to liquid states, $\varepsilon=-2.5 \times 10^{-10}$, dashed lines to glassy states, $\varepsilon=2.5 \times 10^{-10}$. Various forces $F_{\mathrm{ex}}$ are shown with $\delta=\left(F_{\mathrm{ex}}-F_{\mathrm{ex}}^{\mathrm{c}}\right) / F_{\mathrm{ex}}^{\mathrm{c}}=10^{-n}, n=0,1,2,3$, 4 , and 5 as labeled. (For the glass, only $n=0$ and $n=1$ are shown.) Dash-dotted and dotted lines indicate the corresponding host $\beta$-correlator $|G(t)|$, for the liquid respectively the glass, in the panels showing the real parts. For the imaginary part, dotted lines indicate the critical law of the host correlator, $G(t) \sim h\left(t / t_{0}\right)^{-a}$, and $h_{c, j}^{s} / \lambda \approx 3.985 / \lambda$ times this critical law.

chosen here. The $\varepsilon<0$ curve also exhibits von Schweidler's law, $\phi(\hat{t})-f^{c} \sim-B \hat{t}^{b}$ at large rescaled times $\hat{t}=t / t_{\sigma}$. With the distance parameter chosen here, we get $\sigma=6.7 \times 10^{-11}$ and consequently $t_{\sigma}=8.07 \times 10^{14}$; the parameter $B$ is tabulated 28 and estimated as $B=0.68$.

For forces far from the critical threshold, e.g., $\delta=-1$, Eq. 20 is valid. This is demonstrated by the $n=0$ curves in Fig. 6 that correspond to the force-free case: the probe-particle correlation functions closely following the host-liquid curves over all time, up to a fixed amplitude. Evaluation of Eq. (20) in this case yields $\underline{h}_{c}^{s} \approx(0.39,0,0.39)^{\top}$, which is easily verified in the figure. 
In this sense, the tagged-particle dynamics is governed by the dynamics of the host liquid. This well known fact is the basis for various approximations relating singleparticle motion and collective dynamics close to the glass transition. For $n=1$, we get $\underline{h}_{c}^{s} \approx(3.99,2.83,2.54)^{\top}$, and this is also verified by Fig. 6 .

Approaching $F_{\mathrm{ex}}^{\mathrm{c}}$, shown by curves with $\delta=-10^{-n}$ closer to zero (larger $n$ ) in Fig. 6, the coupling found for $\delta=0$ breaks down. Most obviously, in the regime of the von Schweidler law, $\hat{t} \gg 1$, the curves for $n \neq 0$ decay to zero more rapidly than the host correlator, indicated by a plateau in Fig. 6 (dashed lines). This regime will be discussed later.

More subtly, for $\hat{t} \ll 1$, the probe correlators for $F_{\text {ex }} \neq 0$ still appear proportional to the host liquid correlator, but with a different prefactor than the one given in Eq. (20). For $n=5$, Eq. 20 yields $\underline{h}_{c}^{s} \approx$ $(4.16,3.98,2.05)$, not compatible with the asymptotes seen in Fig. 6. We address this now, discussing the limit $\delta \rightarrow 0$ of Eq. (18).

At the critical force, $\delta=0$, we have $\operatorname{det} \underline{A}^{s, c}=0$ and concomitantly $\lim _{\delta \rightarrow 0} \underline{f}_{c}^{s}=\underline{f}_{c}^{s, c}=0$, as discussed above. In that case, also det $\underline{\underline{L}}_{c}^{\bar{s}_{c} c}={ }_{0} c$, rendering Eq. (19) singular and Eq. 20 invalid. In leading order in $\delta, \underline{g}_{\sigma}^{s}(t)$ must be in the kernel of $\operatorname{det} \underline{A}^{s, c}$. In our model, this matrix is only simply degenerate, and we denote by $\underline{h}^{s, c}$ a corresponding null-eigenvector. Keeping the ansatz that $\underline{g}_{\sigma}^{s}(t)$ depends linearly on $g_{\sigma}(t)$, we modify Eq. 20a,

$$
\underline{g}_{\sigma}^{s}(\hat{t})=\underline{h}^{s, c} g_{\sigma}(\hat{t})+\mathcal{O}\left(c_{\sigma}, \delta\right), \quad \delta \rightarrow 0 .
$$

Differentiating Eq. (9) with respect to $\delta$ at $\delta=0$, again using that $\tilde{\tilde{f}}^{s, c}=0$, we find $\underline{\underline{\tilde{A}}}^{s, c}(d / d \delta) \underline{\tilde{f}}^{s, c}=0$ and hence $(d / d \delta) \tilde{f}^{s, c}=\eta \underline{h}^{s, c}$ with some constant $\eta$. Here and in the following, expressions like $d / d \delta f^{s, c}$ are to be read as $\lim _{\delta \rightarrow 0} d / d \delta \underline{f}^{s}$.

Accounting for the next-to-leading order terms in Eq. (18) and using $\tilde{f}_{j}^{s, c}=0$, we get from taking the derivative with respect to $\delta$

$$
\begin{aligned}
& 0=\left(\hat{z} \hat{g}_{\sigma}(\hat{z})\right) \delta \times \\
& \times\left\{\frac{d \underline{\underline{\tilde{A}}}^{s, c}}{d \delta} \underline{h}^{s, c}-\eta \tilde{f} \underline{\mathcal{D}}^{s, c}\left[\underline{h}^{s, c}, \underline{h}^{s, c}\right]+\eta \underline{\mathcal{C}}^{s, c}\left[\underline{h}^{s, c}, \underline{\phi}_{0}^{s}\right]\right\} \\
& +c_{\sigma}\left\{\underline{\mathcal{C}}^{s, c}\left[\underline{h}^{s, c}, \underline{\phi}_{0}^{s}\right] \widehat{z} \widehat{g_{\sigma}^{2}}(\hat{z})+\tilde{f} \underline{\mathcal{C}}^{s, c}\left[\underline{h}^{s, c}, \underline{h}^{s, c}\right]\left(\hat{z} \hat{g}_{\sigma}(\hat{z})\right)^{2}\right\} \\
& +\mathcal{O}\left(c_{\sigma}^{2},\left(c_{\sigma} t_{\sigma}\right)^{-1}, \delta^{2}, c_{\sigma} \delta\right)
\end{aligned}
$$

In the following, we will suppress the indication of omitted higher-order terms as far as they are already denoted here. The term containing $(d / d \delta) \underline{\tilde{A}}^{s, c}$ can be eliminated in favor of $\underline{\mathcal{C}}^{s, c}$ by looking at the Taylor expansion of Eq. 9, which yields

$$
\begin{aligned}
0=\underline{\underline{\tilde{A}}}^{s, c} \cdot \frac{1}{2} \frac{d^{2} \underline{\tilde{f}}^{s, c}}{d \delta^{2}}+\eta \frac{d \underline{\underline{\tilde{A}}}^{s, c}}{d \delta} & \cdot \underline{h}^{s, c} \\
& -\tilde{f} \eta^{2} \underline{\mathcal{C}}^{s, c}\left[\underline{h}^{s, c}, \underline{h}^{s, c}\right] .
\end{aligned}
$$

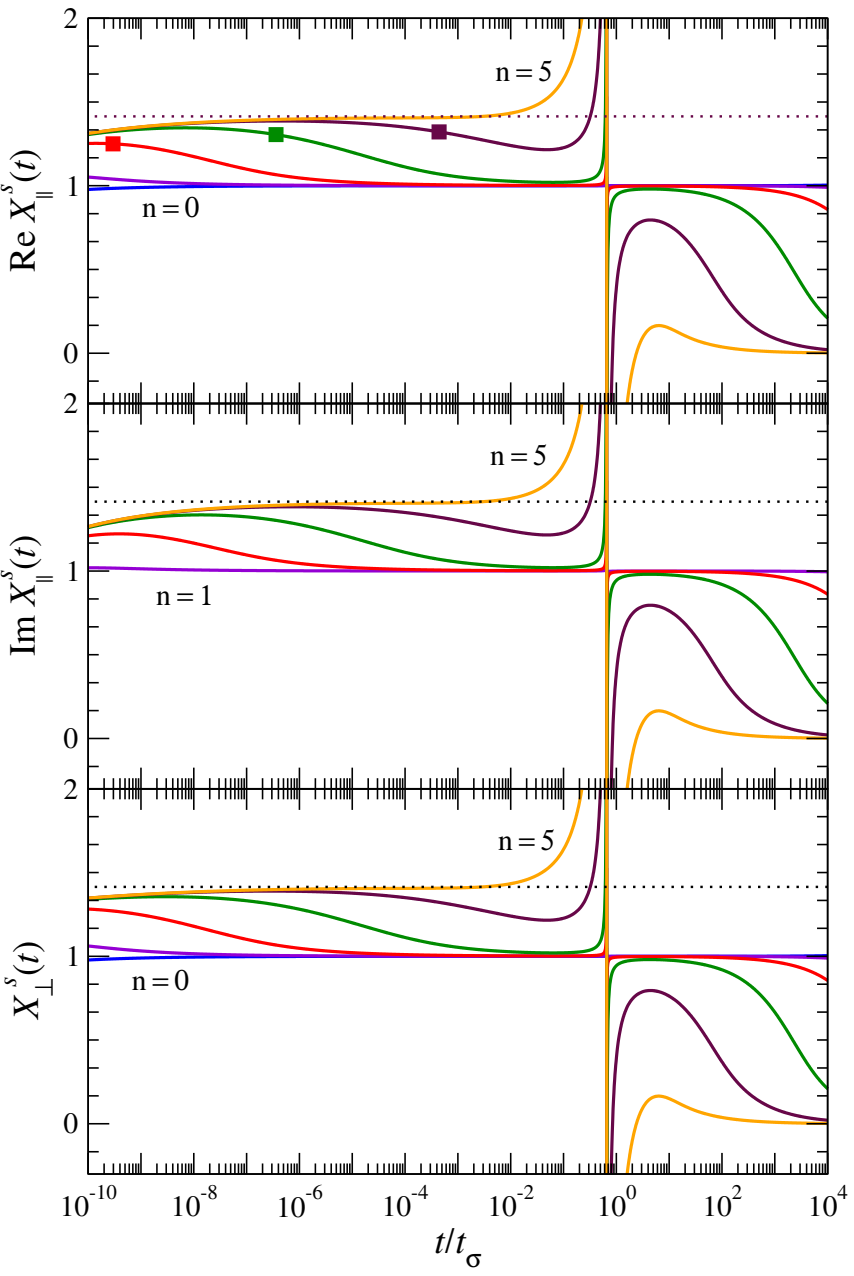

FIG. 7. Normalized ratio of probe-correlation functions to that of the host liquid, $X_{j}(t)=\left[\phi_{j}^{s}(t)-\tilde{f}_{j}^{s}\right] /[\phi(t)-\tilde{f}] /\left(u_{j}^{s, c} A_{\delta}\right)$, cf. Eq. 25, as a function of $\hat{t}=t / t_{\sigma}$. Parameters are chosen as in Fig. 6. only liquid curves, $\varepsilon=-2.5 \times 10^{-10}$ are shown, for $\delta=-10^{-n}$ with $n=0,1,2,3,4$, and 5 (indicated by the labels). The dotted line indicates $A_{\varepsilon} / A_{\delta}=\lambda=\sqrt{2}$. Squares in the upper panel indicate the time scale $t_{\delta}$.

Multiplying with the left null-eigenvector $\underline{\hat{h}}^{s, c}$ of $\underline{\tilde{A}}^{s, c}$, the first term vanishes, and inserting into Eq. (22) provides

$$
\begin{aligned}
0=(\hat{z} \hat{g}(\hat{z})) & \eta \delta\left\{\underline{\hat{h}}^{s, c} \underline{\mathcal{C}}^{s, c}\left[\underline{h}^{s, c}, \underline{\phi}_{0}^{s}\right]-\hat{f} \underline{\hat{h}}^{s, c} \underline{\mathcal{C}}^{s, c}\left[\underline{h}^{s, c}, \underline{h}^{s, c}\right]\right\} \\
+ & c_{\sigma}\left\{\underline{\hat{h}}^{s, c} \underline{\mathcal{C}}^{s, c}\left[\underline{h}^{s, c}, \underline{\phi}_{0}^{s}\right] \widehat{z} \widehat{g}_{\sigma}^{2}(\hat{z})\right. \\
& \left.+f \underline{\tilde{f}}^{s, c} \underline{\mathcal{C}}^{s, c}\left[\underline{h}^{s, c}, \underline{h}^{s, c}\right]\left(\hat{z} \hat{g}_{\sigma}(\hat{z})\right)^{2}\right\}
\end{aligned}
$$

Let us discuss the relevant limits of Eq. (24). We assume that $\underline{\mathcal{C}}^{s, c}\left[\underline{h}^{s, c}, \underline{h}^{s, c}\right] \neq 0$, which is generically the case. Note that in writing Eq. (21), the normalization of the eigenvector $\underline{h}^{s, c}$ is left undetermined. In the limit $\varepsilon \rightarrow 0$, this normalization is now fixed by requiring the first bracket in Eq. (24) to vanish. If we introduce through $\underline{h}^{s, c}=A \underline{u}$ the normalized eigenvector $\underline{u}$ and the 
amplitude $A=\left\|\underline{h}^{s, c}\right\|$, we obtain

$$
A_{\delta}=\frac{\underline{\hat{h}}^{s, c} \cdot \underline{\mathcal{C}}_{c}^{s, c}\left[\underline{u}, \underline{\phi}_{0}^{s}\right]}{f_{c} \underline{\hat{h}}^{s, c} \cdot \underline{\mathcal{C}}^{s, c}[\underline{u}, \underline{u}]}
$$

where the subscript recalls that this result is valid for taking the limit $\delta \rightarrow 0$ after $\varepsilon \rightarrow 0$. The amplitude $A_{\delta}$ does not depend on the actual path along which the hypersurface of delocalization transitions is crossed.

Taking $\delta \rightarrow 0$ first, we require the second bracket in Eq. (24) to vanish. Letting $\varepsilon \rightarrow 0$ after that, we can further make use of Eq. (16) to arrive at

$$
A_{\varepsilon}=\frac{\underline{\hat{h}}^{s, c} \cdot \underline{\mathcal{C}}_{c}^{s, c}\left[\underline{u}, \underline{\phi}_{0}^{s}\right]}{\lambda f_{c} \underline{\hat{h}}^{s, c} \cdot \underline{\mathcal{C}}^{s, c}[\underline{u}, \underline{u}]}=\frac{A_{\delta}}{\lambda} .
$$

Equations (25) and (26) together with Eq. 21) imply that one recovers both the critical power law, $\underline{g}_{ \pm}^{s}(\hat{t}) \sim$ $\hat{t}^{-a}$, and the von Schweidler law, $g_{-}^{s}(\hat{t}) \sim-\hat{t}^{b}$, in the double limit $\varepsilon \rightarrow 0$ and $\delta \rightarrow 0$. The ratio of the correlation functions, however, depends on the order in which this double limit is taken. To exemplify this, define the ratio $X_{j}^{s}(t)=\left(\phi_{j}^{s}(t)-\tilde{f}_{j}^{s}\right) /\left([\phi(t)-\tilde{f}] A_{\delta} u_{j}\right)$. Results for typical parameters are shown in Fig. 7. According to Eqs. (25) and (26), the ratio approaches unity for $\hat{t} \rightarrow \infty$ at not too small $\delta$. This is demonstrated by the $n=0$ curve in the figure. Lowering $\delta \rightarrow 0$, one notices a second plateau in $X_{j}^{s}(t)=1 / \lambda$ that becomes more pronounced for smaller $|\delta|$. For Fig. 7, the result is only valid for $t<t_{\sigma}$. The ranges of validity of the various scaling predictions will be discussed below.

Another scaling result emerges if instead of Eq. 21) we consider the case that $g_{\sigma}(\hat{t})$ is of higher order and can be set to zero in Eq. (18). We will identify a time scale where this is admissible below. Requiring the leading order in Eq. (18) to vanish again leads to the requirement $\underline{\hat{g}}_{\sigma}^{s} \propto \underline{h}^{s, c}$, the null-eigenvector of $\underline{\underline{A}}^{s, c}$. The expansion Eq. (22) reduces to

$$
\begin{aligned}
& 0=\delta\left\{\frac{d \underline{\underline{\tilde{A}}}^{s, c}}{d \delta} \cdot \hat{z} \underline{\hat{g}}_{\sigma}^{s}(\hat{z})-\tilde{f} \mathcal{D}^{s, c}\left[\frac{d \underline{\tilde{f}}^{s, c}}{d \delta}, \hat{z} \hat{\underline{g}}_{\sigma}^{s}(\hat{z})\right]\right\} \\
& +c_{\sigma} \tilde{f} \underline{\mathcal{C}}^{s, c}\left[\hat{z} \hat{g}_{\sigma}^{s}(\hat{z}), \hat{z} \underline{\hat{g}}_{\sigma}^{s}(\hat{z})\right]+\mathcal{O}\left(c_{\sigma}^{2},\left(c_{\sigma} t_{\sigma}\right)^{-1}, \delta^{2}, c_{\sigma} \delta\right)
\end{aligned}
$$

The limit $\varepsilon \rightarrow 0$ is not meaningful in this equation, since the first bracket then only admits the trivial solution. Letting, however, $\delta \rightarrow 0$, we gain $\underline{\mathcal{C}}^{s, c}\left[\hat{z} \underline{\hat{g}}_{\sigma}^{s}(\hat{z}), \hat{z} \underline{g}_{\sigma}^{s}(\hat{z})\right]=$ 0 , which is consistent if we set

$$
\underline{g}_{\sigma}^{s}(\hat{t})=C \hat{t}^{-1 / 2} \underline{h}^{s, c} .
$$

The constant $C$ has to be determined by matching the various asymptotic expansions.

\section{B. Time Scales}

The different results derived above present the asymptotic behavior of the probe-particle correlation functions

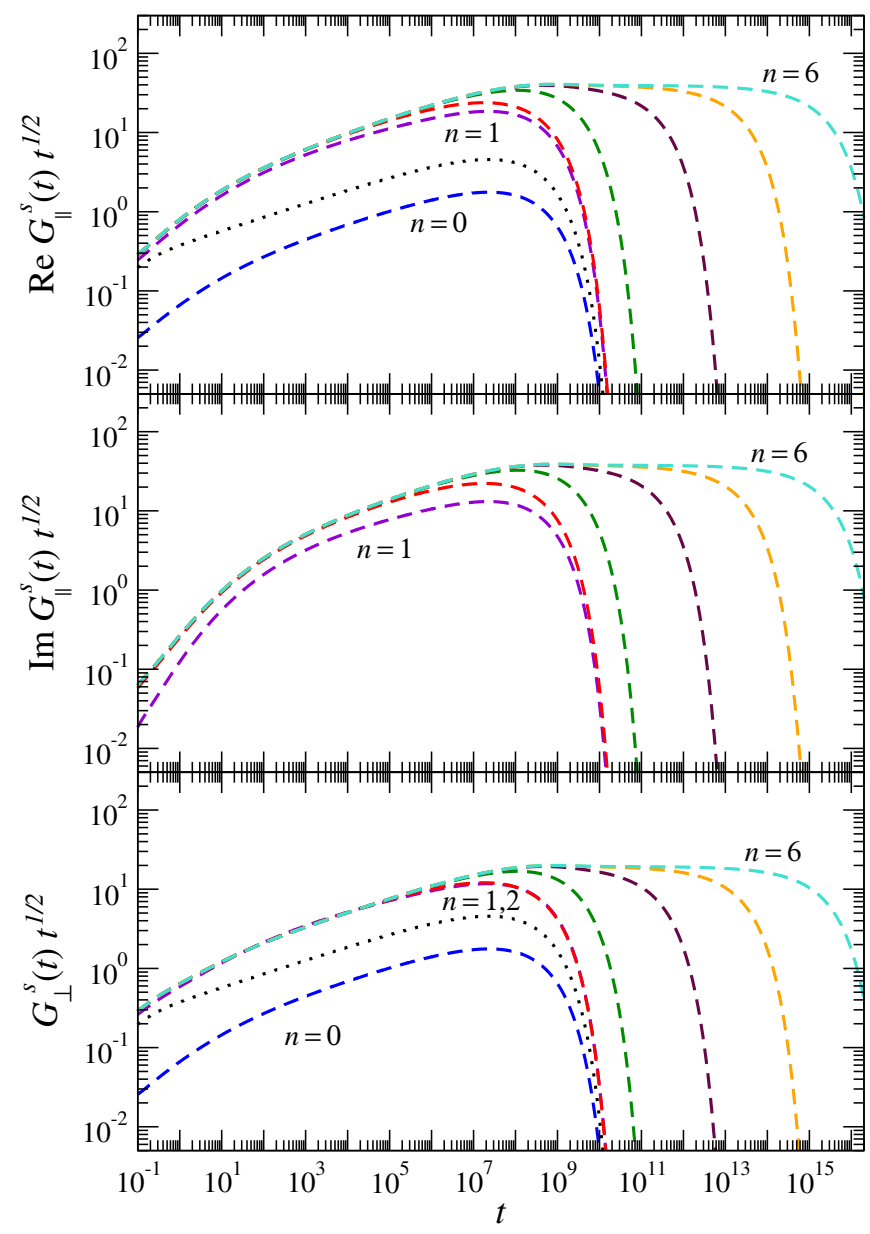

FIG. 8. Correlation functions $\underline{\phi}^{s}(t)-\underline{f}^{s}$ multiplied by $\sqrt{t}$, for $\varepsilon=10^{-6}$, and other parameters as in Fig. 6 Reduced forces are $\delta=-10^{-n}$ with $n=0, \ldots 6$. The dash-dotted line shows the corresponding host-liquid correlator.

in various limits. These correspond to different time scales for which the results hold, which we discuss now. Recall the Laplace transform of a power law $g(t)=t^{-x}$ yields $z \hat{g}(z)=-\Gamma(1-x)(-i z)^{x}$. For short rescaled times, $\hat{t} \rightarrow 0$, the host-liquid $\beta$ correlator assumes the form $g_{\sigma}(\hat{t}) \sim \hat{t}^{-a}$. In Eq. (24), we then identify a time scale $\hat{t}_{\delta}$ that separates the two scaling limits discussed in connection with Eqs. 25) and (26): for $\hat{t} \ll \hat{t}_{\delta}$, the second curly bracket in Eq. (24) dominates, and we recover the $\delta \rightarrow 0$ regime. For $\hat{t} \gg \hat{t}_{\delta}$, the first curly bracket dominates, corresponding to the $\varepsilon \rightarrow 0$ regime. Balancing the power-law exponents, we get $\hat{t}_{\delta}=\mathcal{O}(1)\left|\sigma / \delta^{2}\right|^{1 /(2 a)}$, or

$$
t_{\delta}=\mathcal{O}\left(t_{0}\right)|\delta|^{-1 / a} .
$$

Considering the case where $t_{\delta} \gg t_{\sigma}$, we have to distinguish the two signs of $\varepsilon$. In the glass, $\varepsilon>0, g_{\sigma}$ attains a long time limit of $\mathcal{O}(\sqrt{\sigma})$ so that we may consider Eq. 277) to be valid on a time scale $\hat{t}_{1 / 2}=\mathcal{O}(1)\left|\sigma / \delta^{2}\right|$, or

$$
t_{1 / 2}=\mathcal{O}\left(t_{0}\right)|\sigma|^{1-1 /(2 a)}|\delta|^{-2} .
$$


For times $t \gg t_{1 / 2}$, the trivial solution of Eq. (27) is obtained. Hence the probe $\beta$ correlators $\underline{G}^{s}(t)$ show a nonalgebraic decay to zero for times $t \sim t_{1 / 2}$. Matching this solution, Eq. (28), to the asymptote obtained above, we find that the constant $C$ obeys $C=C^{\prime} \cdot|\sigma|^{1 / 2-1 /(4 a)}$, where $C^{\prime}$ is independent of $\sigma$ and $\delta$. This yields the scaling law

$$
\begin{aligned}
& \underline{G}^{s}(t) \propto|\sigma|^{-1 /(4 a)}|\delta|\left(t / t_{1 / 2}\right)^{-1 / 2}, \\
& t_{\sigma} \ll t \ll t_{1 / 2} .
\end{aligned}
$$

We verify this scaling law in Fig. 8 for $\varepsilon>0$ much larger than considered in Fig. $6, G^{s}(t) \sqrt{t}$ is seen to approach a constant at times long compared to the ultimately exponential relaxation [13] of $G(t)$.

In the liquid, $\varepsilon<0$, we can balance terms of $\mathcal{O}(\delta)$ with those of $\mathcal{O}\left(c_{\sigma}\right)$ inserting the von Schweidler law for the host-liquid $\beta$ correlator, $g_{\sigma}(\hat{t}) \sim-\mathcal{O}(1) \hat{t}^{-b}$, provided that $\hat{t} \gg 1$. As a result, this asymptotic power law can be seen in the probe correlator on a time scale

$$
t_{\sigma, \delta}^{\prime}=\mathcal{O}\left(t_{0}\right)|\delta|^{1 / b}|\sigma|^{-1 /(2 a)-1 /(2 b)} .
$$

Taken together, Eqs. (14), (29), (30), and (32) define five time scales determining the asymptotic behavior of the $\beta$ correlation functions for the probe pulled by an external force. Two of them are inherited from the glasstransition dynamics of the host liquid; $t_{\sigma}$ determines the time scale for the relaxation around the plateau, while $t_{\sigma}^{\prime}$ sets the time scale for the final decay in the liquid. These time scales are marked as diamonds in Fig. 2, we have seen that it suffices to study the correlator $\operatorname{Re} \phi_{\|}^{s}(t)$ in the asymptotic regime, since the other $\phi_{j}^{s}(t)$ are connected via the critical amplitudes $\underline{h}^{s, c}$ to the same timedependent laws.

The time scale $t_{\delta}$ (squares in Fig. 2 separates the regime that is dominated by the proximity to the glass transition $(\sigma \rightarrow 0)$ from the one dominated by the proximity to the delocalization transition $(\delta \rightarrow 0)$. The curve for $\delta=-0.01$ exemplifies the case where $t_{\delta} \ll t_{\sigma}$ so that the dynamics for $t \gg t_{\delta}$ is dominated by the hostliquid glass transition. In this case, the probe-particle $\beta$ correlator is proportional to the host-liquid one, with an amplitude that changes by a factor $1 / \lambda$ at $t \approx t_{\delta}$. For $t \ll t_{\delta}$, Eq. (26) holds for this amplitude, while at $t \gg t_{\delta}$ it drops to the value determined by Eq. (25). This explains the behavior of the correlator ratio $\underline{X}(t)$ shown in Fig. 7. There, squares mark $t_{\delta}$; for the particular cases shown, $t_{\delta}<t_{\sigma}$ holds, so that the ratio for $t>t_{\sigma}$ is given by Eq. 25), viz. $X_{j}(t)=1$, as long as the probe correlators do not decay to zero.

At times $t \gg t_{\sigma} \gg t_{\delta}$, the probe-particle correlator exhibits force-induced decay that couples to the von Schweidler law of the host liquid: this is exemplified for $\delta=-0.01$ in Fig. 2 for $t \approx t_{\sigma, \delta}^{\prime}$ (marked by a triangle). There holds $t_{\sigma, \delta}^{\prime} \propto|\delta|^{1 / b}$, so that approaching the delocalization threshold, $\delta \rightarrow 0$, this force-induced decay is accelerated according to a non-trivial power law.
The curve for $\delta=10^{-5}$ in Fig. 2 on the other hand exhibits the case $t_{\delta} \gg t_{\sigma}$, and the dynamics for $t \ll t_{\delta}$ is dominated by the probe-delocalization limit. In this case, the time scale $t_{1 / 2}$ becomes relevant (marked by a circle in the figure), and the probe correlator decays according to a power law.

Both $t_{\delta}$ and $t_{1 / 2}$ diverge as the force threshold is approached, $\delta \rightarrow 0$, while the host-liquid time scales $t_{\sigma}$ and $t_{\sigma}^{\prime}$, as well as the time scale for which the probe motion couples to the host's von Schweidler law, $t_{\sigma, \delta}^{\prime}$, diverge for $\varepsilon \rightarrow 0$, i.e., upon approaching the glass transition. From the power laws, one infers the relevant scaling combination: for $\left|\sigma / \delta^{2}\right| \gg 1$, the dynamics is determined by the force-induced delocalization, while for $\left|\sigma / \delta^{2}\right| \ll 1$, the glass-transition dynamics describes the relaxation.

\section{THE $\alpha$-SCALING LAW}

In the liquid, the decay of the host correlation function from the plateau towards zero can be discussed on the time scale $t_{\sigma}^{\prime}$ by another scaling law, known as the $\alpha$ scaling law. There, a master function can be derived for the limit $\sigma \rightarrow 0$ in the form [15]

$$
\phi(t) \asymp F\left(t / t_{\sigma}^{\prime}\right) .
$$

The function $F$ is independent of $\sigma$. As a result, the final relaxation of the correlators displays a superposition principle, allowing the long-time part of the correlators to be scaled to a master curve by scaling time with $t_{\sigma}^{\prime}$. We derive a similar law for the probe correlators $\phi^{s}(t)$.

More formally, we introduce $\sigma$-independent scaling correlators $F$ and $\underline{F}^{s}$ by

$$
F(\bar{t}):=\lim _{\varepsilon \rightarrow 0^{-}} \phi(t), \quad \underline{F}_{\delta}^{s}(\tilde{t}):=\lim _{\varepsilon \rightarrow 0^{-}} \underline{\phi}^{s}(t),
$$

with reduced times $\bar{t}=t / t_{\sigma}^{\prime}$ and $\tilde{t}=t / t_{\sigma, \delta}^{\prime}$. From Eqs. (6), we obtain after taking the scaling limit $\varepsilon \rightarrow 0$ with $t_{\sigma}^{\prime}, t_{\sigma, \delta}^{\prime} \rightarrow \infty$ :

$$
\begin{aligned}
\hat{F}(\bar{z}) & =\hat{\mathcal{F}}^{c}[F](\bar{z}) \cdot(1+\bar{z} \hat{F}(\bar{z})), \\
\underline{\hat{F}}_{\delta}^{s}(\tilde{z}) & =\underline{\mathcal{C}}_{c}^{s}\left[\widehat{F \underline{F}_{\delta}^{s}}(\tilde{z}), \tilde{z} \underline{\hat{F}}_{\delta}^{s}(\tilde{z})+\underline{\phi}_{0}^{s}\right] .
\end{aligned}
$$

Here, $\mathcal{F}[F]$ denotes the memory kernel of the host-liquid model, evaluated with the scaling correlator. The initial conditions for Eqs. (35) are obtained by matching them to the long-time limit of the $\beta$-correlation regime in the liquid, i.e. the von Schweidler law. Hence, $F(0)=f_{c}$, and $\lim _{\bar{t} \rightarrow 0}\left(F(\bar{t})-f_{c}\right) \bar{t}^{-b}=-B$. This leads to the standard $\alpha$-scaling regime discussed in detail earlier [15]. Likewise, we identify $\underline{F}_{\delta}^{s}(0)=f_{c}^{s}$ and $\lim _{\tilde{t} \rightarrow 0}\left(\underline{F}_{\delta}^{s}(\tilde{t})-\underline{f}_{c}^{s}\right) \tilde{t}^{-b}=$ $-B^{s}$ where $B^{s}$ is trivially related to $B$ via the critical amplitudes. As the plateau value $\underline{f}_{c}^{s}$ vanishes linearly with $\delta$ upon approaching the delocalization transition, it is reasonable to introduce the probe-particle $\alpha$-scaling correlator

$$
\underline{F}^{s}(\tilde{t}):=\lim _{\delta \rightarrow 0^{-}} \underline{F}_{\delta}^{s}(\tilde{t}) /|\delta|,
$$


to which corresponds the $\alpha$-scaling law

$$
\lim _{\delta \rightarrow 0^{-}} \lim _{\varepsilon \rightarrow 0} \underline{\phi}^{s}(t) /|\delta|=\underline{F}^{s}\left(t / t_{\sigma, \delta}^{\prime}\right) .
$$

It remains to be shown that $\underline{F}^{s}(\tilde{t})$ indeed is independent on $\sigma$ and $\delta$. To see this, expand Eq. (35b),

$$
\begin{aligned}
0 & =\underline{\underline{A}}_{c}^{s, c} \cdot \tilde{z} \underline{\hat{F}}^{s}(\tilde{z}) \\
& +|\delta|\left\{-\frac{d \underline{\underline{A}}_{c}^{s, c}}{d \delta} \cdot \tilde{z} \underline{\hat{F}}^{s}(\tilde{z})+f_{c} \underline{\mathcal{C}}_{c}^{s, c}\left[\tilde{z} \underline{\hat{F}}^{s}(\tilde{z}), \tilde{z} \underline{\hat{F}}^{s}(\tilde{z})\right]\right. \\
& \left.-B^{s} \underline{\mathcal{C}}_{c}^{s, c}\left[\tilde{z} \widetilde{\tilde{t}}^{b} \underline{F}^{s}(\tilde{z}), \underline{\phi}_{0}^{s}\right]\right\}+\underline{\underline{A}}_{c}^{s, c} \cdot \mathcal{O}(\delta) .
\end{aligned}
$$

This suggests the ansatz $\underline{F}^{s}(\tilde{t})=F^{s}(\tilde{t}) \underline{h}_{c}^{s, c}+\mathcal{O}(\delta)$, and multiplying Eq. (37) with the left-null-eigenvector $\underline{\hat{h}}_{c}^{s, c}$ yields an equation that is independent on $\delta$ :

$$
\begin{aligned}
0=-\tilde{z} \hat{F}^{s}(\tilde{z}) \underline{\hat{h}}_{c}^{s, c} \cdot \frac{d \underline{\underline{A}}_{c}^{s, c}}{d \delta} \cdot \underline{h}_{c}^{s, c} \\
+f_{c}\left(\tilde{z} \hat{F}^{s}(\tilde{z})\right)^{2} \hat{\underline{h}}_{c}^{s, c} \cdot \underline{\mathcal{C}}_{c}^{s, c}\left[\underline{h}_{c}^{s, c}, \underline{h}_{c}^{s, c}\right] \\
\quad-B^{s} \tilde{z} \widetilde{t}^{b} F^{s}(\tilde{z}) \underline{\hat{h}}_{c}^{s, c} \cdot \mathcal{C}_{c}^{s, c}\left[\underline{h}_{c}^{s, c}, \underline{\phi}_{0}^{s}\right] .
\end{aligned}
$$

Consequently, $\underline{F}^{s}$ is invariant under rescaling of both variables $\delta$ and $\varepsilon$. It depends, however, on the direction of the path crossing the delocalization transition with respect to the transition hypersurface.

The scaling property of the probe correlators as a function of $\delta \rightarrow 0^{-}$in the limit $\varepsilon \rightarrow 0$ is exhibited by Fig. 9 . Here, some very small $\varepsilon<0$ has been chosen, and the correlation functions of the probe particle are plotted as $\phi_{\alpha}^{s}(\tilde{t}) /|\delta|$ for various $\delta<0$. Reducing $|\delta|$, the $\alpha$-scaling regime is entered, as seen for the $n=2$ through $n=5$ curves: as functions of rescaled time $\tilde{t}=t / t_{\sigma, \delta}^{\prime}$, all the correlators agree with the scaling function $F_{\alpha}^{s}(\tilde{t})$ at long times. The latter shows an initial plateau, followed by a relaxation on a time scale $\tilde{t}=\mathcal{O}(1)$. Qualitatively, this scaling is similar to the usual $\alpha$-relaxation scaling law one finds without external force upon varying $\varepsilon \rightarrow 0$. There, however, the discontinuous nature of the MCT transition predicts that asymptotically, the plateau seen in the $\alpha$ correlator is constant, while for the delocalization transition approached as $\delta \rightarrow 0$, the plateau scales with the distance $|\delta|$ to the transition point. For $n=0$ and $n=1$, the distance $|\delta|$ to the delocalization transition is too large, and the scaling prediction of Eq. (36) no longer holds, as it requires both $\delta \rightarrow 0$ and $\varepsilon \rightarrow 0$. The curve for $n=6$ also shown in Fig. 9 corresponds to the regime $\left|\delta^{2} / \sigma\right| \ll 1$, and no longer satisfies the requirement $\varepsilon \rightarrow 0$ in Eq. (36); it hence deviates again from the scaling law.

\section{THE FRICTION COEFFICIENT}

\section{A. Above the Delocalization Threshold}

We now focus on a discussion of the friction coefficient $\Delta \zeta$ in the asymptotic regime. Let us start by considering

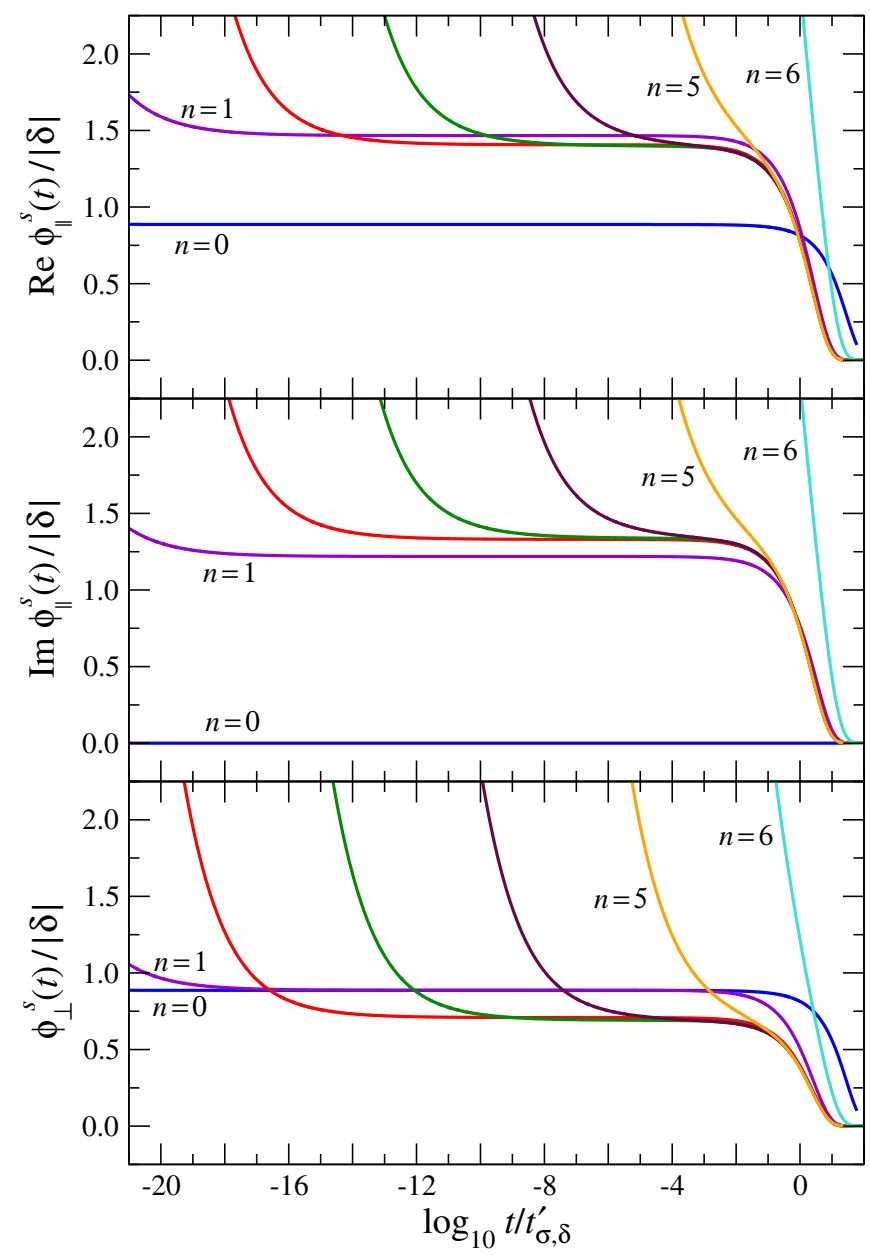

FIG. 9. Test of the $\alpha$-sclaing law for the probe-particle correlation functions: the $\phi_{\alpha}^{s}(t) /|\delta|$ are shown as functions of rescaled time $\tilde{t}=t / t_{\sigma, \delta}^{\prime}$. Parameters are chosen as in Fig. 1 . with $\varepsilon=-10^{-12}$. Reduced forces are given by $\delta=-10^{-n}$, and $n=0,1,2,3,4,5$, and 6 as labeled.

$\delta>0$, i.e., a force exceeding the delocalization threshold, so that even in the glass, $\Delta \zeta<\infty$.

Figure 10 shows the friction coefficients from Fig. 3 in a double-logarithmic representation as a function of the distance to the delocalization threshold, $\delta>0$. At large $\delta$, the qualitative features discussed before in conjunction with Fig. 3 are recovered: a plateau as $\delta \rightarrow \infty$, and an intermediate decay for $\delta \ll 1$ that we will identify with a power law. For $\delta \ll \sqrt{|\sigma|}$, this power law crosses over: for liquid states, the curves approach a constant, since the friction at the critical force $(\delta=0)$ is finite there. For glassy states, the friction diverges as $\delta \rightarrow 0^{+}$, but with a different power law than the one observed for $\delta \gg \sqrt{|\sigma|}$.

Consider first the regime dominated by the $\delta \rightarrow 0$ limit: here, Eq. 28 describes the power-law decay of $\underline{G}^{s}(t) \sim t^{-1 / 2}$ for $\sigma>0$, valid in a time window $\bar{t}_{\sigma} \ll t \ll t_{1 / 2}$. Inserting into Eq. (4), one obtains $\Delta \zeta \approx \int_{\mathcal{O}\left(t_{\sigma}\right)}^{t_{1 / 2}} C^{\prime}|\sigma|^{1 / 2-1 /(4 a)} \cdot\left(t_{0} / t\right)^{1 / 2} \cdot f$ which results 


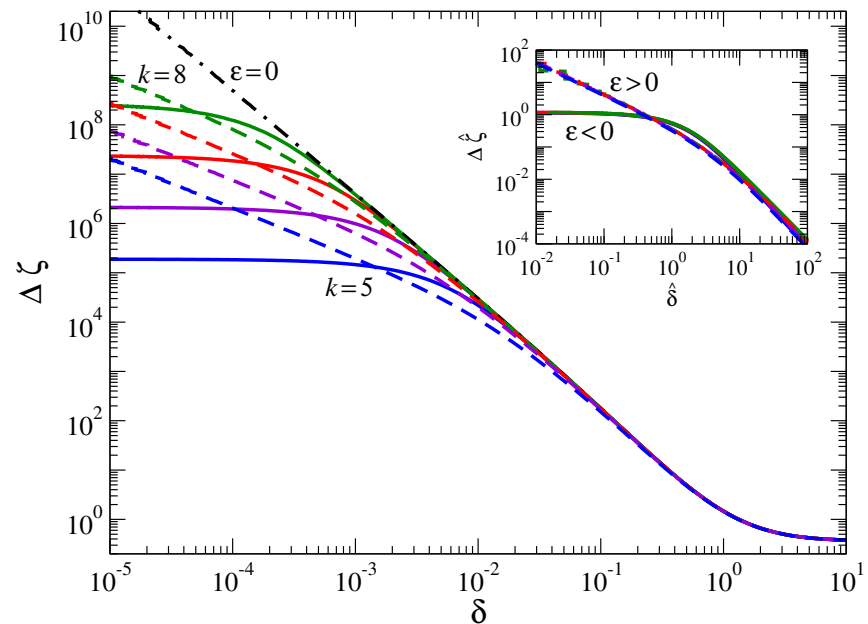

FIG. 10. Friction coefficient increment $\Delta \zeta=\zeta\left(F_{\mathrm{ex}}\right)-\zeta_{0}$ for a probe particle pulled with external force $F_{\text {ex }}$, as a function of the distance to the delocalization threshold, $\delta=$ $\left(F_{\mathrm{ex}}-F_{\mathrm{ex}}^{\mathrm{c}}\right) / F_{\mathrm{ex}}^{\mathrm{c}}$, for $\delta>0$ and various distances to the glass transition $\varepsilon= \pm 10^{-k}$ with $k=5,6,7$, and 8 as labeled. Dashed lines are in the glass $(\varepsilon>0)$, solid lines in the liquid $(\varepsilon<0)$. The curve at the glass transition, $\varepsilon=0$, is shown as a dash-dotted line. Inset: curves for $\varepsilon \neq 0$, as a scaling plot, $\Delta \hat{\zeta}=\Delta \zeta /|\varepsilon|^{1 / 2-1 /(2 a)}$ versus $\hat{\delta}=\delta /|\varepsilon|^{1 / 2}$.

in

$$
\Delta \zeta(\delta) \propto|\sigma|^{1-1 /(2 a)} \cdot \delta^{-1}, \quad \sigma>0,\left|\delta^{2} / \sigma\right| \ll 1 .
$$

In the liquid state, the $t^{-1 / 2}$ power law does not appear. For $\delta \sim \sqrt{|\sigma|}$ we get the $\delta$-independent result in the liquid,

$$
\Delta \zeta(\delta) \propto|\sigma|^{1 / 2-1 /(2 a)}, \quad \sigma<0,\left|\delta^{2} / \sigma\right| \ll 1 .
$$

Equations 39 explain the qualitative behavior seen for $\delta \rightarrow 0$ in Fig. 10

For $t_{\delta} \ll t_{\sigma}$, the probe $\beta$ correlator $\underline{G}^{s}(t)$ for times $t_{0} \ll t \ll t_{\sigma}$ follows the power law $\left(t_{0} / t\right)^{a}$. Integration of Eq. (4) leads to

$$
\Delta \zeta \propto \delta^{-(1-a) / a}, \quad\left|\delta^{2} / \sigma\right| \gg 1,
$$

where the proportionality constant is independent of $\sigma$. This power law is seen in Fig. 10 in the intermediate- $\delta$ regime, where $\delta \gg \sqrt{|\sigma|}$ but still small.

Having identified these power laws, a scaling prediction is obtained for the friction coefficient, for forces just above the threshold. Introducing $\hat{\delta}=\delta / \sqrt{|\varepsilon|}$ and $\Delta \hat{\zeta}=\Delta \zeta /|\varepsilon|^{1 / 2-1 /(2 a)}$, we obtain two master curves, one for $\varepsilon>0$, and one for $\varepsilon<0$. This is shown in the inset of Fig. 10. The scaling curve for the glass shows two power laws, $\Delta \hat{\zeta} \sim \hat{\delta}^{-1}$ for $\hat{\delta} \ll 1$, and $\Delta \hat{\zeta} \sim \hat{\delta}^{1-1 / a}$ for $\hat{\delta} \gg 1$. For our model, $a \approx 1 / 3$, so that the exponent governing the large- $\hat{\delta}$ decay in $\Delta \hat{\zeta}$ is close to 2 , as seen by inspection of Fig. 10. One has to note that the identification of these power laws in experiment or

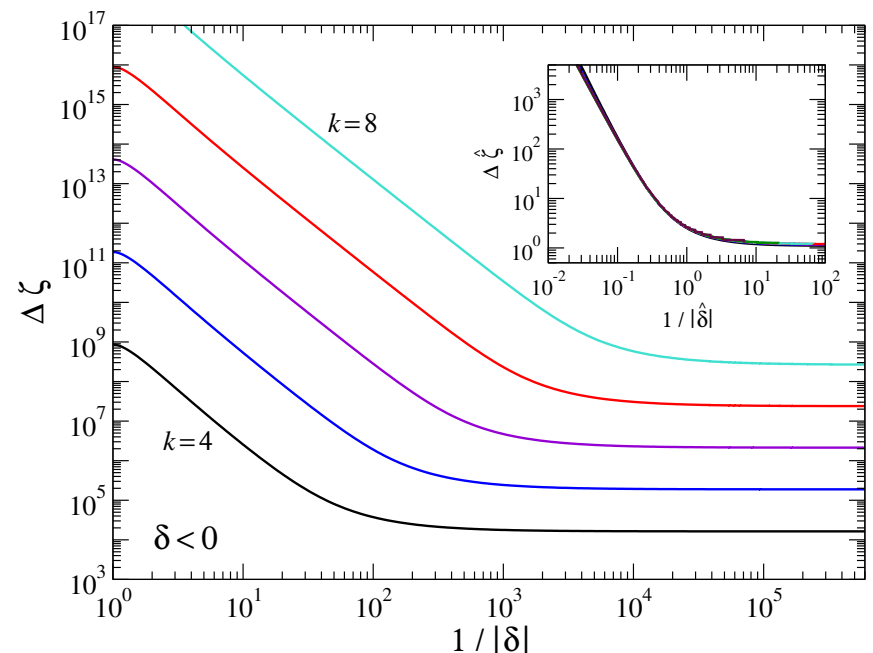

FIG. 11. Friction coefficient increment $\Delta \zeta$ as a function of the distance to the delocalization threshold $\delta$, for $\delta<0$ (i.e., $\left.F_{\text {ex }}<F_{\text {ex }}^{\mathrm{c}}\right)$ in the liquid. The distance to the glass transition is chosen as $\varepsilon=-10^{-k}$, with $k=4,5,6,7$, and 8 as labeled. Inset: scaling plot $\Delta \hat{\zeta}$ versus $\hat{\delta}$ for the same data and also including $k=9$ and $k=10$.

simulation is hampered by the fact that one has to approach both $\varepsilon \rightarrow 0$ and $\delta \rightarrow 0$. For finite distances to the transition, preasymptotic corrections quickly become dominant. Fits using the schematic model we discuss here have to be performed with varying coupling coefficient $v^{s}$ [10. Compared to the case displayed by Fig. 10 . this introduces a regular shift of the curves [29].

\section{B. Below the Delocalization Threshold}

We now discuss the case $\delta<0$. Here, the probe remains localized within glassy states, so that for $\delta<0$ and $\sigma>0, \Delta \zeta=\infty$ holds trivially. For $\sigma<0$, the final $\alpha$ relaxation will give the dominant contribution to the friction coefficient, which is obtained by inserting the $\alpha$-scaling law into Eq. (4),

$$
\begin{aligned}
\Delta \zeta \approx(1-\mu) \int_{0}^{\infty} & F\left(t / t_{\sigma}^{\prime}\right) F_{\delta, 1}^{s}\left(t / t_{\sigma, \delta}^{\prime}\right) d t \\
& +\mu \int_{0}^{\infty} F\left(t / t_{\sigma}^{\prime}\right) F_{\delta, 3}^{s}\left(t / t_{\sigma, \delta}^{\prime}\right) d t
\end{aligned}
$$

This expression yields a factorization of $\Delta \zeta$ of the form $\Delta \zeta \sim|\sigma|^{-1 /(2 a)-1 /(2 b)} Z(\delta)$ for $\sigma \rightarrow 0$, where $Z$ is universal. If $t_{\sigma, \delta}^{\prime} \gg t_{\sigma}$, i.e., $\left|\delta^{2} / \sigma\right| \gg 1$, and additionally $|\delta| \ll 1$, we further use that $\underline{F}_{\delta}^{s}\left(t / t_{\sigma, \delta}^{\prime}\right)$ is proportional to $|\delta|$. From the von Schweidler law we then arrive at

$$
\Delta \zeta \propto|\sigma|^{-1 /(2 a)-1 /(2 b)}|\delta|^{1 / b+1}, \quad \sqrt{|\sigma|} \ll|\delta| \ll 1 .
$$

Hence, the exponent of von Schweidler's law is also present in the behavior of $\Delta \zeta$, as can bee seen in Fig. 11 , 
where $\Delta(\zeta)$ is plotted as a function of $1 /|\delta|$ for forces below the delocalizaton threshold, in the liquid. Note that for $F_{\text {ex }} \rightarrow 0$, i.e., $\delta \rightarrow-1$, the von Schweidler regime is cut off due to the microscopic relaxation of the correlation functions, and $\Delta \zeta\left(F_{\mathrm{ex}}=0\right)-\Delta \zeta \sim F_{\mathrm{ex}}^{2}$ is obtained as mentioned further above. As the glass transition is approached, this window of initial linear response shrinks.

Again, Eq. (42) includes a scaling prediction: plotting $\Delta \hat{\zeta}$ as a function of $\hat{\delta}$, where the rescaled variables are defined above, the curves for all $\varepsilon$ close to the glass transition can be scaled onto one master curve. This is shown in the inset of Fig. 11. The von Schweidler law is seen for $\hat{\delta} \ll 1$, in the form $\Delta \hat{\zeta} \sim \hat{\delta}^{1 / b+1}$. This exponent is close to 2.6 for the model parameters chosen here, as one can verify in the figure.

\section{Large-Force Plateau}

We come briefly back to the case, where the probe particle is delocalized. For $\delta \gg \mathcal{O}(1)$, the $\beta$-scaling regime does not give the dominant contribution to $\Delta \zeta$ any longer and the short-time dynamics of the correlation functions is more important. In this case it is sufficient to consider the probe dynamics alone and that of the host liquid as essentially arrested, i.e., $v_{j}^{s} \phi(t) \approx v_{j \text {,eff }}^{s}$ as constants. The friction coefficient for $\delta \rightarrow \infty, \Delta \zeta_{\infty}=\Delta \zeta\left(F_{\text {ex }} \rightarrow \infty\right)$ can then be calculated as

$$
\begin{array}{r}
\Delta \zeta_{\infty} \approx \phi_{0} \int_{0}^{\infty} d t\left\{(1-\mu) \operatorname{Re} \phi_{\|}^{s}(t)+\mu \phi_{\perp}^{s}(t)\right\} \\
=\phi_{0}\left\{(1-\mu) \operatorname{Im} \hat{\phi}_{1}^{s}(z=0)+\mu \operatorname{Im} \hat{\phi}_{3}^{s}(z=0)\right\} .
\end{array}
$$

From Eq. 6b), $\underline{\psi}^{s}=\underline{\hat{\phi}}^{s}(z=0)$ fulfills a linear equation system, $\cdot \underline{\psi}^{s}=i \underline{\underline{\omega}}^{-1} \underline{\phi}_{0}^{s}+\underline{\mathcal{C}}^{s}\left[\underline{\psi}^{s}, \underline{\phi}_{0}^{s}\right]$. This yields an algebraic approximation for $\Delta \zeta_{\infty}$. For the model discussed here, we get

$$
\Delta \zeta_{\infty} \sim \mu f_{\text {eff }}+\mathcal{O}\left(1 / F_{\text {ex }}^{2}\right),
$$

where $f_{\text {eff }}=\mathcal{O}(1)$ is the approximately constant value of the host-liquid correlator $\phi(t)$ for the time window over which the probe-particle correlations decay. The increment of the friction coefficient as $F_{\mathrm{ex}} \rightarrow \infty$ hence is given by the parameter $\mu$ that controls the admixture of "perpendicular" modes to the integral determining $\Delta \zeta$. This fact has been exploited in Ref. [10] in order to quantitatively describe the large-force amplitude of measured data. Setting $\mu=0$ results in an expression for $\Delta \zeta$ that decays to zero as $1 / F_{\mathrm{ex}}^{2}$ at large forces. Setting further $v_{2}^{s}=0$, one obtains the model used in Ref. 9], where $\Delta \zeta_{\infty}=f_{\text {eff }}\left(1+v_{\text {eff }}^{s}\right) /\left(1-v_{\text {eff }}^{s}+F_{\text {ex }}^{2}\right)$. This expression holds for $v_{\mathrm{eff}}^{s} \geq 1$, ensuring positivity of the result.

The reason that the correlation function $\phi_{\|}^{s}(t)$ does not contribute to the friction-coefficient increment at large forces, is its oscillatory decay. Fig. 12 shows exemplary results for a very large force $F_{\text {ex }}$ : both $\phi(t)$, shown as dotted lines, and $\phi_{\perp}^{s}(t)$ decay roughly exponentially, with a

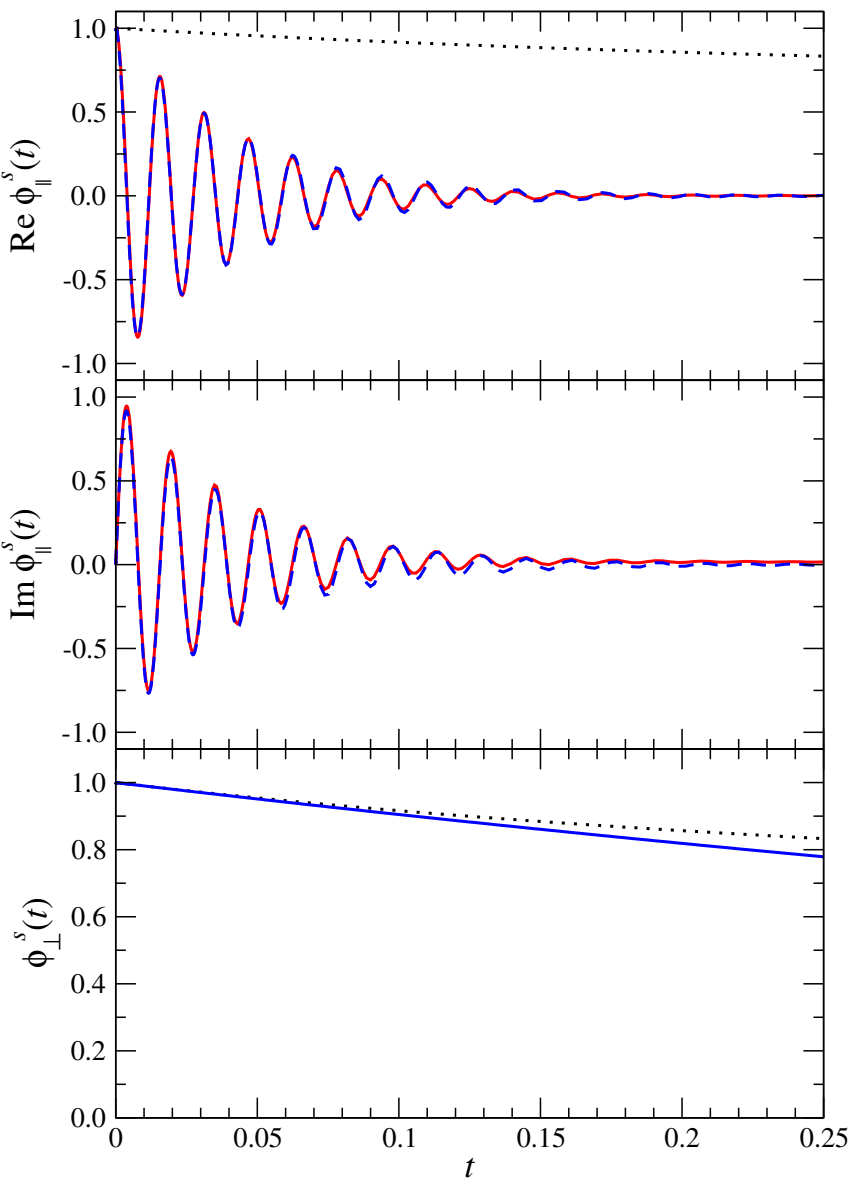

FIG. 12. Correlations functions $\phi_{\|}^{s}(t)$ and $\phi_{\perp}^{s}(t)$ of the schematic model, with parameters as in Fig. 1] but a large external force, $F_{\mathrm{ex}}=402.7$. Full lines show the numerical solutions of the model, dashed lines the least-square fits using an exponentially damped function $\phi_{\|}^{s}(t)=e^{-\nu t} e^{i\left(F_{\mathrm{ex}}+\xi\right) t}$ with parameters $\nu=22.6$ and $\xi=-2.1$. The host-liquid correlator $\phi(t)$ is shown as a dotted line in the top and bottom panels.

relaxation time that is much larger than the one relevant for $\phi_{\|}^{s}(t)$ in this regime. The latter becomes proportional to a damped oscillation, $\phi_{\|}^{s}(t) \approx \exp \left[-t+i \kappa_{\|} F_{\text {ex }} t\right]$ in the absence of any memory-kernel damping, cf. Eq. 3a. Taking into account the remaining damping from the memory kernel, we find that $\phi_{\|}^{s}(t)$ for large $F_{\text {ex }}$ is nicely fitted with $\exp [-\nu t] \exp \left[i\left(\kappa_{\|} F_{\text {ex }}+\xi\right) t\right]$. Such a fit is included in Fig. 12, with parameters $\nu=22.6$ and $\xi=-2.1$ determined by a least-square error minimization; it is virtually indistinguishable from the numerical result for $\phi_{\|}^{s}(t)$ in the figure.

The microscopic interpretation of these oscillations is a steady motion of the probe, $\underline{r}^{s}(t)=\underline{r}^{s}(0)+\underline{v} t$, leading to $\phi_{q}^{s}(t)=\exp [\underline{i q} \cdot \underline{v} t]$. For $\underline{q} \| \underline{F_{\text {ex }}}$, choosing a wave vector characterizing typical probe-host interaction length scales, we recover $\zeta\left(F_{\text {ex }} \rightarrow \infty\right)=q_{\|} / \kappa_{\|}$. The schematicmodel parameter $\kappa_{\|}$therefore relates typical length scales to the high-force friction coefficient. It is also clearly seen 
that for $\underline{q} \perp F_{\mathrm{ex}}$, no oscillations remain in the correlation function; this is correctly captured in the schematic model.

\section{CONCLUSION}

We have discussed a schematic mode-coupling model for the nonlinear response of the friction coefficient in force-driven active microrheology. Expressions describing the asymptotic behavior of the probe-particle density correlation function in the directions parallel and perpendicular to the force within the schematic model have been derived. From these, together with the known asymptotic behavior of the equilibrium host-liquid correlation function, we have inferred two-parameter scaling laws that yield scaling forms for the probe-particle friction coefficient. The two small parameters are the distance to the glass transition of the host liquid, $\varepsilon$, and the distance $\delta$ to the delocalization transition, where a frozen-in probe becomes mobile in response to a forceinduced local melting of the host. There exists a scaling limit based on the combination $\left|\varepsilon / \delta^{2}\right|$ : if this parameter is small, the external force is a small perturbation to the glassy dynamics of the host liquid, while for $|\delta| \ll \sqrt{|\varepsilon|}$, the vicinity to the force-induced local melting of the glass dominates the dynamics.

The asymptotic expressions for the correlation functions lead to the identification of five relevant time scales: two scales, $t_{\sigma}$ and $t_{\sigma}^{\prime}$, determine the power-law regimes for the host-liquid correlator. These diverge upon approaching the glass transition, $\sigma \rightarrow 0$, and are independent of the external force characterized by $\delta$. For the coupling of the probe correlators to that of the host liquid, a time scale $t_{\delta}$ was identified that diverges as the critical force is approached, $\delta \rightarrow 0$. It separates two regimes of proportionality, $\underline{G}^{s}(t) \propto G(t)$, but with different prefactors. Considering the long-time decay of the probe correlator, we identified two further time scales, $t_{1 / 2}$ and $t_{\sigma, \delta}^{\prime}$, describing, respectively, the decay inside a glassy host liquid and the coupling of the probe relaxation to the host's von Schweidler law. For the friction coefficient, the various asymptotic regimes of the correlation function induce several power-law regimes around the critical threshold force, $F_{\mathrm{ex}}^{\mathrm{c}}$, marking the point where the external force becomes more effective in breaking cages than thermal fluctuations.

In the linear response regime, the well-known asymptotic result of equilibrium MCT is recovered, stating that the structural relaxation of the probe is, up to a wavevector dependent amplitude, given by the relaxation of the host, cf. Eq. 20. In a sense, this is the justification of the discussion of microrheology response in terms of generalized Stokes-Einstein relations, where one tries to relate the diffusivity of the probe particle to the collective dynamics of the surrounding host (expressed by its shear viscosity). Within MCT, a relation of the form $D \sim 1 / \eta$ is the consequence of Eq. 20, with prefactors that are typically of the size expected from the Stokes-Einstein relation 34. Note, however, that the true Stokes-Einstein relation is of the form $D \sim k_{\mathrm{B}} T / \eta$, where the factor $k_{\mathrm{B}} T$ identifies it as a relation connecting a macroscopic transport coefficient to microscopic fluctuations. Indeed, recent experiments indicate that the factor $k_{\mathrm{B}} T$ is absent in the empirical relation between $D$ in $\eta$ found in viscous liquids [35], in agreement with Eq. 20.

Small external forces will preserve this coupling, but as the critical force is approached, the range of validity of Eq. 20 continuously shrinks. The initial deviation from the linear reponse regime, by symmetry, has to be quadratic, $\Delta \zeta\left(F_{\mathrm{ex}}\right)-\Delta \zeta\left(F_{\mathrm{ex}}=0\right) \sim-F_{\mathrm{ex}}^{2}$. Approaching $F_{\text {ex }}^{\mathrm{c}}$, however, the von Schweidler law governs the force thinning behavior, i.e., a power law involving the exponent $b$ is seen in $\Delta \zeta$. This is, however, a small effect, not immediately apparent from experimental data.

The delocalization transition is, within the present schematic model, identified as a continuous transition, i.e. the nonergodicity parameter of the probe-particle density correlators vanishes continuously. In a microscopic model, this would correspond to a continuous broadening of the probability distribution function for the probe position. This can be understood as a singleparticle localization length that diverges due to the applied force. However, in the schematic model, no such interpretation is obvious, since the model does not carry any information related to large length scales. It is also expected that the exponents found in the asymptotic analysis carried out here will be modified by taking into account long-wavelength fluctuations 30.

Above the threshold force, tracer motion is always delocalized. For this case, the friction coefficient increment $\Delta \zeta$ follows two power laws: a trivial force thinning with exponent -1 is predicted in the glass, valid for very small $\left|F_{\text {ex }}-F_{\text {ex }}^{c}\right|$. This law is, closer to the glass transition, replaced by a power law involving the MCT critical exponent, i.e., force thinning with exponent $y=1-1 / a<-1$. Inserting typical values found for hard-sphere like systems within MCT, $a \approx 1 / 3$, one gets for the force thinning exponent $y \approx-2$.

Considering still larger forces, $F_{\mathrm{ex}} \rightarrow \infty$, a feature seen in experiment is a second plateau in $\Delta \zeta>0$, indicating that the friction experienced by the pulled particle is not just the solvent friction. Although hydrodynamic interactions (HI) in the colloidal suspension will play a major role in this regime [32, the second plateau is not only due to them, as it is also found in Brownian-dynamics simulations 9] that do not include HI. Within the schematic model, the additional contribution can be traced back to the correlation function $\phi_{\perp}^{s}(t)$ mimicking the density correlators in the direction perpendicular to the force.

Although the schematic model we have discussed gives a reasonable quantiative description of the friction coefficients, and a qualitative one for intermediate-lengthscale density correlation functions, the question remains whether the force-driven delocalization of a probe particle in the glass is continuous in the sense mentioned 
above. Another possibility is that of a discontinuous local yielding, where the plateau of the tagged-particle correlation function $f^{s}$ does not decrease to zero as $F_{\text {ex }} \rightarrow F_{\text {ex }}^{\mathrm{c}}-0$, but to some nonzero constant (so that a discontinuous jump in the nonergodicity parameter at $F_{\text {ex }}^{c}$ results). These scenarios are not easily distinguished following the behavior of the friction coefficient. Correlation functions from computer simulation of Brownian soft-sphere systems [9, 10, appear compatible with the continuous scenario incorporated in the schematic model discussed here. However, recent MD simulations of the mean-squared displacement in active microrheology show a plateau that does not change appreciably with varying $F_{\text {ex }}$ 31, and thus may point to a discontinuous transition scenario. Note that even the connection between schematic and "full" microscopic MCT remains unclear at the moment. This connection hinges upon the assumption that the two modes chosen for the probe correlator in the schematic model are sufficient to represent the full set of critical modes in the bifurcation transition of the full model. In this respect, the microscopic model of Ref. 9] is an extension of MCT that is qualitatively different from other extensions of the original MCT where it could be shown that the latter's bifurcation class $\mathcal{A}_{l}$ was not left.

\section{ACKNOWLEDGMENTS}

We thank M. Fuchs and C. Harrer for discussions. This work was supported by DFG SFB-Transregio TR6, project A7. Th.V. thanks for funding through the Helmholtz-Gemeinschaft (HGF VH-NG 406), and the Zukunftskolleg der Universität Konstanz. M.V.G. thanks for funding through the research initiative Analysis and Numerics of Evolution Equations with Applications in the Sciences of the Universität Konstanz and the graduate school IMPRS of the Max Planck Institute for Mathematics in the Sciences.
[1] T. A. Waigh, Rep. Prog. Phys. 68, 685 (2005).

[2] T. M. Squires, Langmuir 24, 1147 (2008).

[3] A. Erbe, M. Zientara, L. Baraban, C. Kreidler, and P. Leiderer, J. Phys.: Condens. Matt. 20, 404215 (2008).

[4] C. Wilhelm, Phys. Rev. Lett. 101, 028101 (2008).

[5] M. B. Hastings, C. J. Olson Reichhardt, and C. Reichhardt, Phys. Rev. Lett. 90, 098302 (2003).

[6] P. Habdas, D. Schaar, A. C. Levitt, and E. R. Weeks, Europhys. Lett. 67, 477 (2004).

[7] S. R. Williams and D. J. Evans, Phys. Rev. Lett. 96, 015701 (2006).

[8] T. M. Squires and T. G. Mason, Annu. Rev. Fluid Mech. 42, 413 (2010).

[9] I. Gazuz, A. M. Puertas, Th. Voigtmann, and M. Fuchs, Phys. Rev. Lett. 102, 248302 (2009).

[10] M. V. Gnann, I. Gazuz, A. M. Puertas, M. Fuchs, and Th. Voigtmann, Soft Matter 7, 1390 (2011).

[11] W. Götze and L. Sjögren, Z. Phys. B 65, 415 (1987).

[12] M. Fuchs and M. E. Cates, Faraday Discuss. 123, 267 (2003).

[13] W. Götze and L. L. Sjögren, J. Math. Analysis Appl. 195, 230 (1995).

[14] T. Franosch and Th. Voigtmann, J. Stat. Phys. 109, 237 (2002).

[15] W. Götze, Complex Dynamics of Glass-Forming Liquids (Oxford University Press, 2009).

[16] M. Fuchs and M. E. Cates, J. Rheol. (NY) 53, 957 (2009).

[17] D. Hajnal and M. Fuchs, Eur. Phys. J. E 28, 125 (2009).

[18] M. Krüger, F. Weysser, and M. Fuchs, Eur. Phys. J. E 34, 88 (2011).

[19] M. Fuchs and M. E. Cates, Phys. Rev. Lett. 89, 248304 (2002).
[20] L. Sjögren, Phys. Rev. A 33, 1254 (1986).

[21] G. Nägele, Phys. Rep. 272, 215 (1996).

[22] Th. Voigtmann, EPL 96, 36006 (2011).

[23] T. M. Squires and J. F. Brady, Phys. Fluids 17, 073101 (2005).

[24] I. C. Carpen and J. F. Brady, J. Rheol. 49, 1483 (2005).

[25] D. Widder, The Laplace Transform (Princeton University Press, 1972), 8th ed.

[26] V. Arnol'd, Catastrophe theory (Springer, Berlin, 1992), 3rd ed.

[27] W. Götze, in Liquids, Freezing and Glass Transition (Les Houches, Session LI), edited by J. P. Hansen, D. Levesque, and J. Zinn-Justin (North-Holland, Amsterdam, Oxford, New York, 1991).

[28] W. Götze, J. Phys.: Condens. Matter 2, 8485 (1990).

[29] M. V. Gnann, Analysis of schematic models of modecoupling theory for colloids in external fields, Diploma thesis, Universität Konstanz (2009).

[30] S. K. Schnyder, F. Höfling, T. Franosch, and Th. Voigtmann, J. Phys.: Condens. Matt. 23, 234121 (2011).

[31] D. Winter, J. Horbach, P. Virnau, and K. Binder, Phys. Rev. Lett. 108, 028303 (2012).

[32] A. S. Khair and J. F. Brady, J. Fluid Mech. 557, 73 (2006).

[33] J. M. Brader, Th. Voigtmann, M. Fuchs, R. G. Larson, and M. E. Cates, Proc. Natl. Acad. Sci. USA 106, 15186 (2009).

[34] M. Fuchs and M. R. Mayr, Phys. Rev. E 60, 5742 (1999).

[35] J. Brillo, A. I. Pommrich, and A. Meyer, Phys. Rev. Lett. 107, 165902 (2011). 